\title{
Two-Dimensional CdSe-Based Nanoplatelets: Their Heterostructures, Doping, Photophysical Properties, and Applications
}

This article reviews the use of quasi-2-D CdSe-based colloidal quantum wells (nanoplatelets), covering their heterostructures, doping opportunities, and extraordinary photophysical properties, and extending the review to their applications in light-emitting diodes, lasers, and luminescent solar concentrators.

By Manoj Sharma ${ }^{\circledR}$, SAVAs Delikanli ${ }^{\circledR}$, ANd Hilmi Volkan Demir ${ }^{\circledR}$

ABSTRACT I In the past decade, colloidal quantum wells, also known as 2-D semiconductor nanoplatelets (NPLs), have been added to the colloidal nanocrystal (NC) family. Through the unique control of the thickness with monolayer precision, these novel materials exhibit strong 1-D quantum confinement that offers unique optical properties along with the possibility of fabricating advanced heterostructures, which are not possible with other quantum-confined nanostructures. The 2-D CdX ( $X=\mathrm{Se}, \mathrm{S}$ )-based NPLs provide high color purities, fast fluorescence lifetimes, and large exciton binding energies. This review covers the latest developments in the successful utilization of these flat NCs in different nanophotonic device applications. The synthesis of the advanced heterostructures of flat 2-D NCs (e.g., core-shell, core-crown, and core-crown-shell) has

Manuscript received April 28, 2019; revised July 24, 2019 and September 17, 2019; accepted September 20, 2019. Date of publication October 18, 2019; date of current version April 28, 2020. This work was supported in part by the National Research Foundation, Prime Minister's Office, Singapore, through the Investigatorship Program under Grant NRF-NRFI2016-08 and in part by the Singapore Agency for Science, Technology and Research (A*STAR) SERC Pharos Program under Grant 15273 00025. (Manoj Sharma and Savas Delikanli contributed equally to this work.) (Corresponding author: Hilmi Volkan Demir.) The authors are with the Luminous! Centre of Excellence for Semiconductor Lighting and Displays, School of Electrical and Electronic Engineering and Division of Physics and Applied Physics, School of Physical and Mathematical Sciences, Nanyang Technological University, Singapore 639798, and also with the Department of Electrical and Electronics Engineering and the Department of Physics, UNAM-Institute of Materials Science and Nanotechnology, Bilkent University, 06800 Ankara, Turkey (e-mail: hvdemir@ntu.edu.sg).

Digital Object Identifier 10.1109/JPROC.2019.2944277 matured very rapidly, and new exciting optical and electronic applications are emerging. Doping of these atomically thin NCs also offers new possibilities for their utilization in different solar light harvesting, magnetic, electronic, and lasing applications. This review also includes the recent advancements in the understanding of their unique optical properties that are of utmost importance for their practical implementation in light-emitting devices and lasers. Finally, we present a future perspective on their successful utilization in different nanophotonic applications.

KEYWORDS | Colloidal quantum wells (CQWs); doping; heterostructures; nanophotonic devices; Semiconductor nanoplatelets (NPLs); 2-D materials.

\section{IN T R O D U C TION}

In the early 1980s, the discovery of quantum size effects in semiconductor nanocrystals (NCs) and the resulting size-dependent optical properties began a new branch of research for materials scientists, chemists, and physicists [1], [2]. Later, in 1993, the synthesis of monodispersed spherical CdSe NCs using colloidal methods was reported [3]. Soon after this report, different experimental groups using similar colloidal methods started to synthesize and analyze different semiconductor materials at the nanoscale (e.g., ZnSe, ZnS, and CdS) [4]-[8]. In the next few years, materials with different compositions, 
configurations (e.g., core/shell, type I, type II, and quasi-type II), and quantum confinements (0-D and 1-D) were reported at the nanoscale [9], [10]. Joo et al. [11] reported the synthesis of 2-D CdSe nanoribbons possessing a wurtzite crystal structure 1-D confinement. Ithurria and Dubertret [12] presented the synthesis of the first 2-D zinc blende (ZB) CdSe colloidal quantum wells (CQWs), also widely known as nanoplatelets (NPLs). Interestingly, they extended this technique to other semiconductor NCs, e.g., CdS and CdTe NPLs, while maintaining precise control of the thickness at the atomic scale [13], [14]. With the addition of these 2-D NPLs to the family of colloidally synthesized NCs, the family became complete in terms of different quantum-confined NCs (e.g., 0-D, 1-D, and 2-D).

The 2-D CdSe NPL-based NCs later added in the last decade have shown interesting optical properties based on their controlled thickness at the atomic scale that in turn lead to narrow emission linewidths, large exciton binding energies, high absorption cross sections, and high oscillator strengths [13]. These exceptional optical properties are all strongly required and were long sought for colloidal NCs due to their possible application in lasing, light emission, and solar energy harvesting. Furthermore, with the knowledge and experience gained by different research groups through the synthesis and handling of colloidal quantum dots (CQDs), these materials can be easily extended to more efficient and stable architectures possessing pure 1-D confinement. In a short time, different heterostructures of atomically flat NCs, e.g., core-shell, core-crown, and corecrown-shell, were reported [15]-[17]. These materials are more efficient and possess excellent optoelectronic properties. Because of the anisotropic shape of NPLs, a different type of heterostructure, namely, "core-crown," is possible. For crown growth, another semiconductor material can be grown on the periphery of CdSe host NPLs. This process implies that the thicknesses of the core and crown region are the same, and both the core and crown regions can separately absorb the illuminated photons, which in turn can increase the absorption cross section per particle [15]. These types of architectures are not possible with other quantum-confined NCs (e.g., 0-D and 1-D). Depending on the choice of crown materials, e.g., CdS, CdTe, or CdSeTe, type-I, type-II, or quasi-type-II heterostructures can be realized. In the last five years, different groups have focused on improving the synthesis of these advanced heterostructures and using them for different applications [14], [16], [18], [19].

Another important class of semiconductor NCs was achieved by the doping of transition metal or rare-earth ions in the host. Doping is a process of intentional introduction of impurity ions into the host semiconductor material. Controlled doping in host semiconductor NCs allows significant modification of their electrical, optical, and magnetic properties. However, doping in CQWs has not been explored to the same extent as CQDs. Only very recently, copper doping in CdSe NPLs has been shown by a high-temperature nucleation-doping method and partial cation exchange (CE) reactions. In 2017, copper doping of flat CdSe NPLs was demonstrated, which shows extraordinarily high photoluminescence (PL) quantum yields (QYs) along with tunable and Stokes-shifted dopant-induced near-infrared (NIR) emission [20]. These materials were shown to perform well as emitters in luminescent solar concentrators (LSCs). Furthermore, controlled doping of atomically flat core and core-shell NPLs with $\mathrm{Cu}, \mathrm{Mn}, \mathrm{Ag}$, and $\mathrm{Hg}$ was shown to lead to improved magnetic, optical, and electronic properties compared to similar 0-D-confined doped CQDs [20]-[25].

Along with the studies performed and the understanding of interesting properties (e.g., narrow PL linewidths, high oscillator strength, and high PL QYs) obtained, in the last few years, successful use of NPLs in different optoelectronic applications [e.g., lasers, light-emitting diodes (LEDs), LSCs, and biolabeling] has been widely reported [18], [20], [26]-[28]. Our group, in particular, focused on the synthesis of advanced heterostructures, doping of CdSe NPLs, and applications of these materials in lasers and LEDs. Two early reviews on this category of NCs were published in 2013 and 2015 [14], [29]. Nevertheless, they focused on either all categories of NPLs (indium-based, copper-based, tin-based, lead-based, zincbased, cadmium-based, and so on) or overall aspects of the flat NCs developed up to that time. The readers are suggested to follow these detailed reviews for understanding different 2-D materials possessing 1-D confinement. However, in the last five years, with the advancements in the synthesis and understanding of their novel photophysical properties, flat CdSe-based NPLs and their complex heterostructures have achieved a unique place among all categories of colloidal NCs. Suppressed Auger recombination due to the 2-D nature, successful doping of atomically thin CdSe NPLs and their unique heterostructures enable these materials to serve as superior materials for nextgeneration lasers, light-emitting materials, efficient photocatalysts, and LSCs. In this review, we will mainly focus on the synthesis updates of colloidally synthesized CdSebased NPLs, including their heterostructures and doping in these flat CdSe NCs. Second, we will focus on the interesting photophysical properties and recent developments in the successful utilization of these flat NCs, particularly in lasing, light-emitting devices, and solar light-harvesting applications. In general, throughout the review unless mentioned, we will compare CdSe NPLs and their heterostructures (core-shell, core-crown, and so on) with similar 0-D CdSe core and CdSe/CdS core-shell CQDs, which have been widely studied for these applications. In the last part, we will discuss the challenges along with a possible outlook for these materials.

\section{C O L L O I D A L 2-D MATERIAL S}

\section{A. CdSe NPLs}

CdSe-based 2-D NPLs with different crystal structures can be synthesized by colloidal methods. Joo et al. [11] 

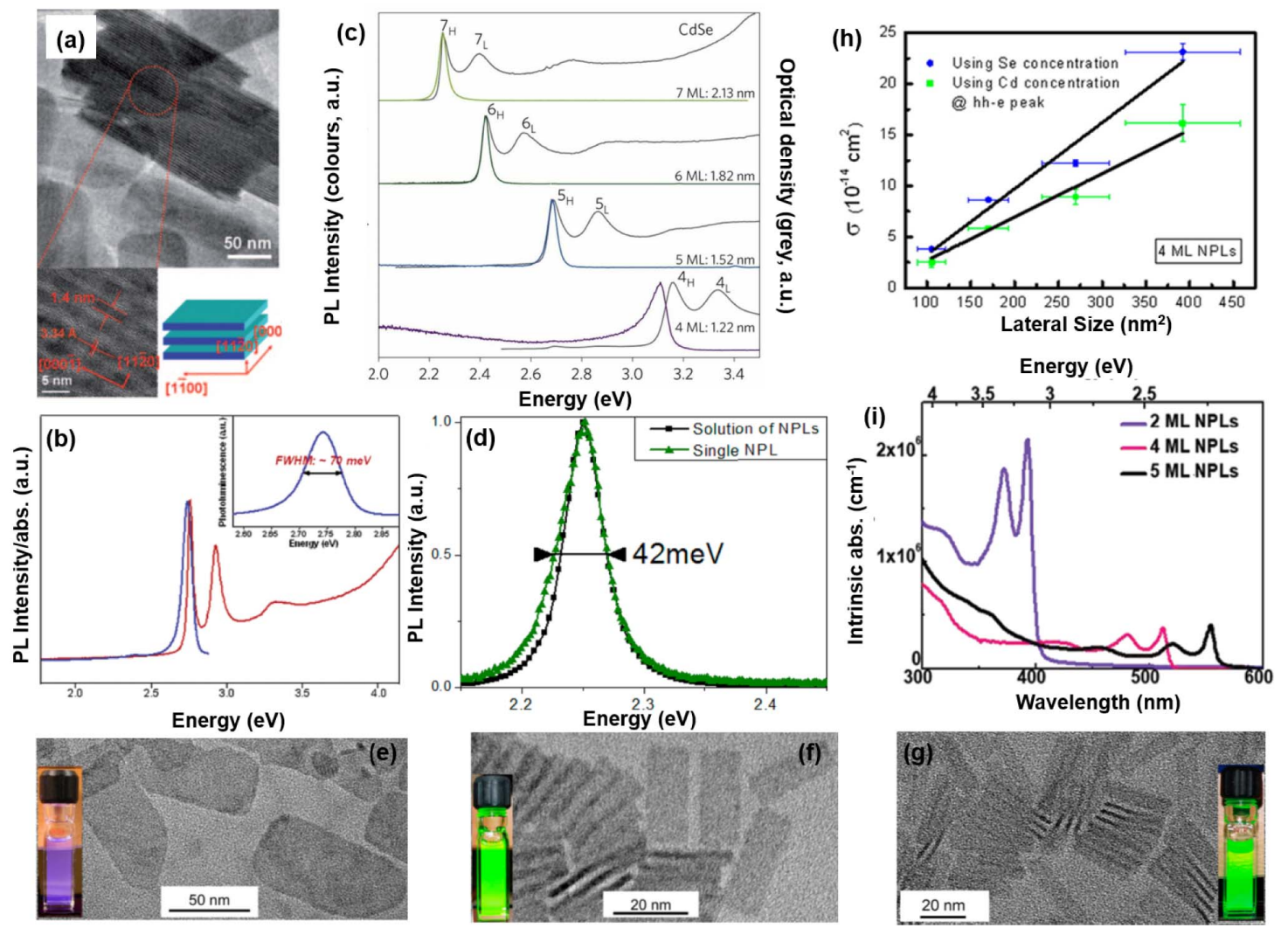

Fig. 1. (a) TEM and HRTEM images of lamellar-structured CdSe nanosheets with a wurtzite crystal structure (top); the bottom left shows the edges of CdSe nanosheets, and the bottom right image illustrates the crystallographic nature of the nanosheets. Reprinted with permission from [30], copyright 2009, Wiley-VCH. (b) UV-vis absorption and room-temperature PL emission spectra of CdSe nanoribbons. Reprinted with permission from [11], copyright 2006, American Chemical Society. (c) PL emission and absorption spectra of CdSe NPLs with the ZB crystal structure and tunable thicknesses. Reprinted with permission from [13], copyright 2011, Springer Nature. (d) PL spectra of 5-ML CdSe NPLs in an ensemble and at the single platelet level. The FWHMs of both cases are similar (42 meV). Reprinted with permission from [34], copyright 2012, American Chemical Society. (e)-(g) TEM images of CdSe NPL colloids with the ZB structure and different thicknesses. Inset: corresponding emission images of CdSe NPLs with 3-5-ML vertical thicknesses. Reprinted with permission from [38], copyright 2015, American Chemical Society. (h) From Inductively coupled plasma optical emission spectrometry (ICP-OES) measurements, the Se and Cd concentrations of different CdSe NPLs were obtained, which helped to calculate the absorption cross section of different 4-ML CdSe NPL samples having varying lateral sizes. Lateral size dependence of the absorption cross section at the energy of hh-e transition peak is shown. Reprinted with permission from [39], copyright 2015, American Chemical Society. (i) Intrinsic absorption spectra of CdSe NPLs having different thicknesses (e.g. for 2, 4, and 5 ML). Reprinted with permission from ref [42], copyright 2019, Wiley-VCH.

reported the synthesis of CdSe nanoribbons with a wurtzite crystal structure. These 2-D materials with a wurtzite crystal structure were synthesized by low-temperaturebased lamellar-like template growth. Fig. 1(a) shows the transmission electron microscopy (TEM) and HRTEM micrographs of CdSe nanoribbons showing a fixed vertical thickness of $1.4 \mathrm{~nm}$ [30]. Fig. 1(b) shows the PL emission and absorption spectra of these CdSe nanoribbons, which shows a narrow emission linewidth with a fullwidth-at-half-maximum (FWHM) of $70 \mathrm{meV}$ along with clear electron/heavy hole and electron/light hole absorption features in the absorption spectra that suggest the formation of 2-D NCs with strong 1-D confinement. Following this approach, other materials, such as CdS and $\mathrm{CdTe}$, were also synthesized [31]. For the synthesis of these nanoribbons, cadmium chloride powder was dissolved in an amine in which a selenium/sulfur/tellurium precursor was slowly reduced [30]. However, the control of the thickness of these nanoribbons was not investigated in detail. Additionally, CdSe NPLs with ZB crystal structures were synthesized in 2008 for the first time [12]. To synthesize these anisotropic-shaped NPLs possessing cubic structures, first, magic-sized seeds were obtained by mixing long-chain Cd(carboxylate) ${ }_{2}$ as a $\mathrm{Cd}$ precursor, which was heated, together with Se powder in a noncoordinating solvent. Furthermore, the addition of short-chain $\mathrm{Cd}$ (carboxylate) ${ }_{2}$ at varying temperature (e.g., $180{ }^{\circ} \mathrm{C}-240{ }^{\circ} \mathrm{C}$ ) led to the formation of 2-D NPLs [13], [29], [32]. In recent years, the addition of short-chain carboxylate was found to be crucial for the synthesis of these anisotropic-shaped NPLs [33]. Furthermore, the thickness of these 2-D platelets can be tailored by varying the addition temperature of the short-chain carboxylate salts. Generally, 3-5 monolayer (ML) CdSe NPLs can be synthesized by these methods [see Fig. 1(c)]. The PL spectra of 5-ML CdSe NPLs in an ensemble and 
at the single platelet level are shown in Fig. 1(d). Clearly, the FWHMs of both cases are similar (42 meV), which shows the absence of any thickness variation among the NPL ensemble [34]. Christodoulou et al. [35] reported the use of cadmium chloride and cadmium oleate at higher temperatures, which can convert the initial 5-ML-thick CdSe NPLs to 8-ML CdSe NPLs. Another important method to tune the thickness of as-synthesized core CdSe NPL is to grow a CdSe, CdS shell. Chu et al. [36] have shown a method to control the thicknesses of CdSe NPLs from 3 to 11 MLs by growing further CdSe MLs on presynthesized 3-4-ML CdSe core NPLs. Recently, Riedinger et al. [33] studied the formation of quasi-2-D NPLs in detail. Briefly, via both experimental and theoretical approaches, they did not observe any evidence of a template growth mechanism, such as that for wurtzite-shaped CdSe NPLs. They emphasized that the solubility of the cadmium precursor plays an important role in obtaining anisotropic shapes from ZB-structured magic-sized CdSe seeds. For details regarding the synthesis and formation of these anisotropicshaped NPLs, the readers are referred to the detailed report [33].

Both ZB- and wurtzite-structured CdSe 2-D NCs possess narrow PL linewidths [see Fig. 1(b) and (c)]. However, the PL QYs of ZB-structured CdSe NPLs are higher than those of hexagonal nanoribbons. Moreover, the thicknesses of these ZB CdSe NPLs can be fine-tuned with atomic-level precision [see Fig. (e)-(g)]. These tunable thicknesses result in a tunable emission in the visible spectrum with extraordinary optical properties, e.g., narrow PL linewidths in the visible region, high absorption cross sections, high oscillator strengths, and ultrahigh optical gains for lasing [13], [35], [37], [38]. Fig. 1 shows the colloidal NPLs synthesized with both wurtzite and ZB crystal structures.

Obtaining the absorption cross section $(\sigma)$ is important for understanding the fundamental optical properties of NPLs. The absorption cross-sectional values also provide a way to calculate the number of electron-hole pairs generated per absorption event. For this purpose, absorption cross-sectional values are an important parameter for using these materials in different nanophotonic applications [39], [40]. Fig. 1(h) shows the experimentally determined absorption cross-sectional values for colloidally synthesized CdSe NPLs (for given vertical thickness as a function of lateral size). The per-particle $\sigma$ values for the 4-ML CdSe NPLs with different areas were determined by using the relation, $\sigma=2303 A /\left(C_{\mathrm{NPL}} N_{\mathrm{A}} L\right)$. Here, $A$ is the absorbance, $N_{\mathrm{A}}$ is the Avogadro's number, and $L$ is the optical path length [41]. Over the whole absorbance spectrum, the absorption cross section increases as a function of the platelet area [see Fig. 1(h)]. Fig. 1(i) shows the intrinsic absorption cross section of CdSe NPLs with different vertical thicknesses. By dividing the obtained absorption cross section by the volume of the NPLs, the intrinsic absorption cross section was obtained [42]. The obtained intrinsic absorption of 2-ML CdSe NPLs $\left(\approx 2.15 \times 10^{6} \mathrm{~cm}^{-1}\right)$ at their first excitonic peak is significantly larger than the intrinsic absorption of thicker (4-5 ML) CdSe NPLs $\left(\approx 4.1 \times 10^{5} \mathrm{~cm}^{-1}\right)$ and CdSe CQDs $\left(\approx 2.8 \times 10^{5} \mathrm{~cm}^{-1}\right)$ [40].

\section{B. Heterostructures}

NPLs, with their anisotropic pure 1-D confinement and narrow emission, are appealing candidates for nextgeneration optoelectronic applications, such as displays and lasers. Regarding solution-processed nanostructures, the electronic and physical properties can be modified and enhanced by the epitaxial growth of a shell layer around the semiconductor core [43]. With the type-I band alignment, the exciton wave function is largely confined in the core, while the type-II band alignment leads to the separation of the charge carriers. In the case of quasi-type-II band alignment, while one of the carriers is localized at the core, the wave function of the other carrier is largely relaxed into the shell. For CQDs, such control of the shell results in enhancement of the QY [43], a reduction in PL emission blinking [44]-[46], and an improvement of the photostability [47]. These enhanced properties have led to increasing interest in the use of heterostructures in LEDs [6], [48], lasers [49], and biological imaging [50]. Unlike CQDs, which are limited by the growth of shell layers, NPLs provide unique opportunities for the design of complex heteroarchitectures due to the opportunity to vertically grow a shell as well as laterally grow a crown. Here, we discuss core/shell NPLs, core/crown NPLs, and advanced NPL heterostructures combining the growth of both shells and crowns as well as multicrowns.

\section{Core-Shell NPLs}

Shells of different compositions have been successfully grown on CdSe NPLs using various approaches to improve the QY and stability and provide new functionalities. First, examples of shell growth were presented by Ithurria and Talapin [37] and Mahler et al. [51] via growth of CdS and CdZnS shells on seed CdSe NPLs at room temperature. A representative TEM image and absorption and PL spectra of core/shell NPLs are presented in Fig. 2(d) and $(\mathrm{h})$, respectively. The excitonic transitions redshifted by more than $100 \mathrm{~nm}$ with the growth of a shell on these NPLs, as shown in Fig. 2(f) and (g), due to the relaxation of the charge carrier wavefunctions into the shell. Ithurria and Talapin [37] demonstrated the growth of CdS shells on CdSe NPLs with atomic layer precision via the technique they called colloidal atomic layer deposition (c-ALD). With this method, the layers of CdS can be deposited on the seed NPLs with atomic layer precision, providing perfect control of the optical properties. Anionic and cationic MLs can be alternatively deposited on seed NPLs using this method. In this method, a sulfide layer was first grown on Cd-rich facets of NPLs using $\mathrm{TMS}_{2} \mathrm{~S},\left(\mathrm{NH}_{4}\right)_{2} \mathrm{~S}, \mathrm{Na}_{2} \mathrm{~S}$, or $\mathrm{NaSH}$, and a cationic layer was grown by the addition of a cadmium salt. Later, this approach was extended to grow CdZnS, CdMnS, and MnS 
(a)

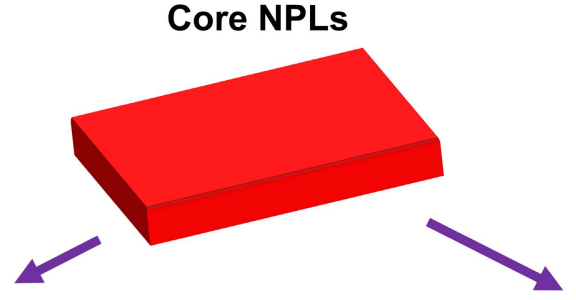

Core/crown NPLs

laterally grown shell)

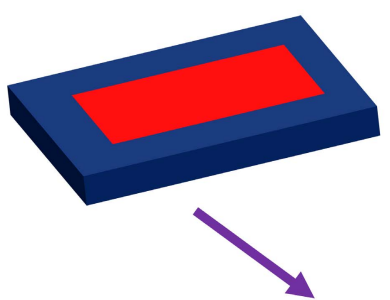

Core/crown/shell NPLs

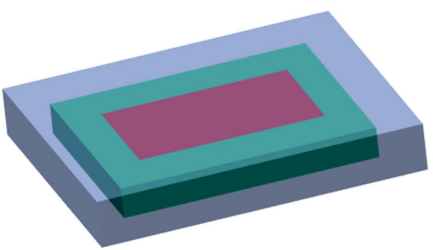

(b)

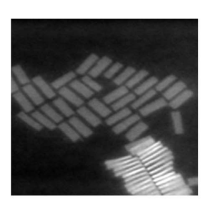

Core/shell NPLs

(vertically grown shell)

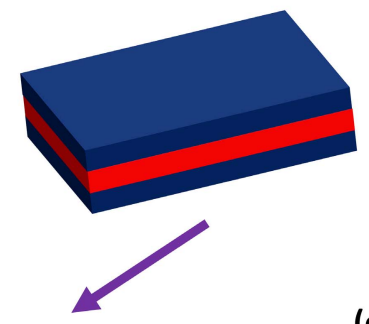

(e)

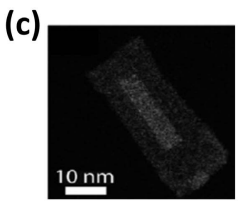

(d)
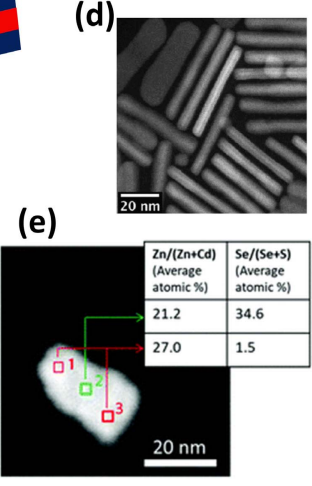

(f)

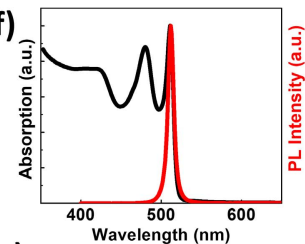

(g)

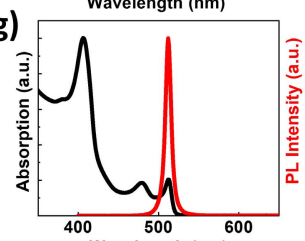

(h)

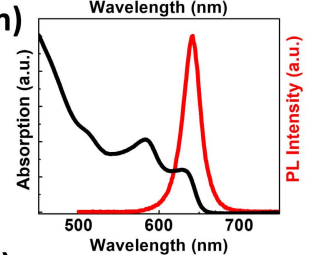

(i)

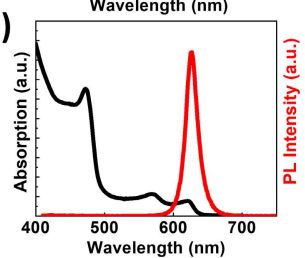

Fig. 2. (a) Schematics of core, core/crown, core/shell, and core/crown@shell NPLs. (b) TEM image of core NPLs. (c) TEM image of core/crown NPLs. Reprinted with permission from [29], copyright 2015, American Chemical Society. (d) TEM image of core/shell NPLs. Reprinted with permission from ref [51], copyright 2012, American Chemical Society. (e) TEM image of core/crown@shell NPLs. Reprinted with permission from [52], copyright 2019, Royal Society of Chemistry. Absorption and PL spectra of (f) core, (g) core/crown, (h) core/shell, and (i) core/crown@shell NPLs.

on CdSe NPLs to extend the control of the optical properties and realize new functionalities [22], [52]-[54]. The approach of Mahler et al. [51] helped realize the continuous growth of CdS and CdZnS shells on CdSe NPLs. With this method, QYs reaching 80\% in nonpolar solutions were reported [55], although this method could not provide precise control of the optical properties, as provided by the c-ALD technique. With the coating of CdSe NPLs with CdS and CdZnS shells, the FWHM of the PL peak increased from 37 to $65 \mathrm{meV}$, which can be attributed to an increase in the electron-phonon coupling with the addition of the shell [55]. The growth of a ZnS shell on CdSe NPLs at moderate temperatures $\left(100{ }^{\circ} \mathrm{C}-150{ }^{\circ} \mathrm{C}\right)$ under ambient atmosphere was also recently achieved [56]. These type-I core-shell NPLs exhibit PL quantum efficiency values up to $60 \%$ and excellent stability under ambient conditions.

The synthesis of core/shell CdSe/CdS and CdSe/ZnS at high temperatures was also reported very recently [57]-[59]. Such high-temperature synthesis methods are required for better crystal quality and optical stability, as previously observed for core/shell CQDs [46]. High-temperature shell growth on CdSe NPLs was first reported by Norris et al. [57], and they extended an approach previously used for CQDs based on the addition of low-reactivity precursors at $\sim 300{ }^{\circ} \mathrm{C}$ [46], [60]. This method makes deposition of thick CdS shells feasible, which was not possible with previously existing low-temperature continuous-growth methods. They obtained uniform and high QY NPLs while preserving the narrow emission (FWHM $\approx 20 \mathrm{~nm}$ ). The growth of a $\mathrm{ZnS}$ shell at high temperatures with low-reactivity precursors (i.e., octanethiol) yielded near-unity efficiency highly stable core/shell NPLs with improved thermal stability [58]. In particular, NPLs with thick shells exhibited excellent thermal stability, which makes them suitable candidates for lasing and LED applications. Optical gain of these NPLs with thick shells should benefit from their large absorption cross section and reduced Auger recombination as a result of the reduced spatial overlap between the electron and hole envelope wave functions, especially in the case of quasi-type-II and type-II core-shell structures [61]-[63]. A reduction in gain threshold was observed in colloidal CQDs with the addition of shell layers [62]-[64]. In addition, their excellent thermal stability after many heating cycles along with their nearunity efficiency and narrow emission makes them excellent candidates for LED applications. Alloy-gradient shells of CdZnS on these CdSe NPLs may further provide better 
optical properties compared with pure CdS and ZnS shells, as demonstrated in core-shell QD systems [60]. These smooth alloy shells may enable further enhancement of the optical gain compared with pure shells of CdS and ZnS.

\section{Core-Crown NPLs}

Lateral growth of crowns on CdSe NPLs provides a tuning approach to control the optical and electronic properties of CQWs while maintaining the same thickness. The first examples of lateral growth of crowns on NPLs were achieved by using an acetate salt on CdSe NPLs [65], [66]. CdS crowns were grown on the seed CdSe NPLs, and the lateral extension of the crowns could be controlled by adjusting the amount of added precursors. A high-resolution TEM image of a CdSe/CdS core/crown NPL is presented in Fig. 2(c). In contrast to core/shell NPLs, in CdSe/CdS core/crown NPLs, the energies of the excitonic transitions of the core NPLs remain the same, as shown in Fig. $2(\mathrm{~g})$, due to the insignificant change in the confinement of the carriers in the lateral dimension with the addition of the crown. The addition of a CdS crown passivates the periphery of the core NPLs and thus increases the PL efficiency of the resulting NPLs. The CdS crown effectively increases the absorption cross section of the NPLs, and excitons generated in the crown region can effectively funnel into the CdSe core as a result of the band offsets and large exciton binding energy. The superior optical properties of quasi-type-II CdSe/CdS core/crown NPLs have led to their use in LEDs and optical gain applications [67], [68].

Inverted type-I core/crown NPL heterostructures with tunable optical properties were also reported by Delikanli et al. [69]. To realize these inverted type-I NPLs, a CdSe crown was grown on the seed CdS NPLs, and by varying the lateral size of the CdSe crown, the emission was tuned over a wide range between the emission wavelengths of the core CdSe and CdS NPLs. In these NPLs, the exciton generated in the CdS crown is also efficiently funneled into the CdSe crown with an adjustable size. NPLs with thin crowns showed reasonable performance in the white light generation, with the color render index (CRI) reaching 80 .

In addition, type-II NPL heterostructures were reported by growing CdTe or CdSeTe crowns on CdSe NPLs [15], [17], [70]. In these type-II heterostructures, the recombination time is orders of magnitude longer than those in the core-only NPLs as a result of the spatial separation of the carriers due to the band offsets [15], [17]. In contrast to core and type-I core/crown NPLs with a quasi-zero Stokes-shifted emission and an extremely narrow emission, the PL emission of these NPLs shows a significant Stokes shift $(>100 \mathrm{~nm})$ due to the type-II emission. The QY of these NPLs can be greatly enhanced by controlling the alloying between Se and Te. QYs reaching 95\% were reported for type-II structures having alloyed CdSeTe crowns [70]. This high QY in these engineered structures was attributed to the large exciton binding energy and large in-plane conductivity in these ultrathin NPLs. In addition, with alloying, the emission wavelength can be tuned over a wide range. These alloyed NPLs with Stokes emission were shown to be promising candidates for LED and lasing applications [18], [71], [72]. These alloyed type-II NPLs showed very low amplified spontaneous emission (ASE) thresholds, high net modal gains, and long gain lifetimes [72].

\section{E. Complex NPL Heterostructures}

The low stability of the optical performance of core NPLs is one of their drawbacks. Recently, the consecutive growth of crowns and shells on CdSe NPLs was demonstrated to significantly improve the QY and optical gain performance compared to core@shell NPLs [16], [52]. These complex heterostructures resembling a particle in a box can be obtained by growing CdZnS shell layers on CdSe/CdS core/crown NPLs. To realize this particle-in-a-box heterostructure, CdZnS layers can be grown on core/crown NPLs with atomic layer precision through the c-ALD technique. A TEM image of such a core/crown@shell heterostructure is shown in Fig. 2(e). The absorption and PL peaks significantly redshift with the growth of a shell, as shown in Fig. 2(i). The absorption cross section of these platelets is further enhanced compared with the simple core/shell structure because of the absorption contributed by the crown region. The obtained core/crown@gradientalloyed shell NPLs routinely exhibit QYs as high as $80 \%$ in nonpolar solvents [52]. Although similar results were reported for core/shell NPLs, core/shell NPLs suffer from reproducibility issues. The QYs of core/crown@gradientalloyed heterostructures can reach $90 \%$ in an aqueous medium [52], which is significantly better than those of core/crown NPLs [73], as a result of passivation of both the surface and periphery with a wide-bandgap shell. The PL and absorption spectra of the core/crown@shell NPLs in a nonpolar solvent and water along with their QYs are shown in Fig. 3(a). In addition, the hydrophobicity and 2-D geometry of these structures were exploited to achieve brightly emitting films of nonstacked oriented NPLs with directed emission. Such selective attachment of NPLs to functionalized surfaces at the nanoscale is shown in Fig. 3(b)-(d). Directed emission via films of functionalized core/crown@gradient-alloyed NPLs is highly advantageous for light extraction in light-emitting platforms. In these films, $>90 \%$ of the dipoles are oriented in-plane, leading to directed emission [52], [74].

The growth of core-multicrown NPLs was shown to be another approach for acquiring increased photostability and better optical gain performance. CdSe/CdSe ${ }_{1-x} \mathrm{Te}_{x} /$ CdS core/multicrown hetero-NPLs were synthesized by growing first $\mathrm{CdSe}_{1-x} \mathrm{Te}_{x}$ and then CdS crowns on CdSe NPLs [75]. In these multicrown NPLs, the addition of the CdS layer decreases the chance of stacking, which 
(a)

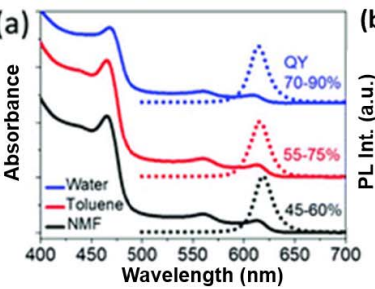

(b)

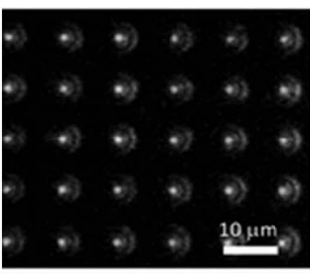

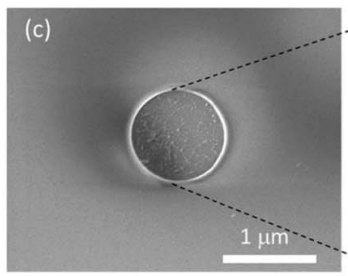

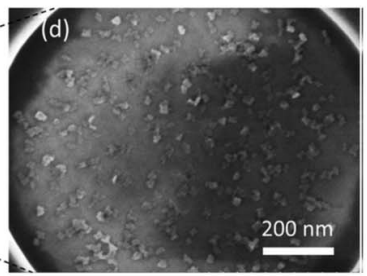

Fig. 3. (a) Absorbance and PL spectra of core/crown/shell NPLs in different solvents. (b) PL intensity map of NPLs for a pattern with 1- $\mu$ m-diameter holes. TEM image of core NPLs. (c) SEM image showing NPLs attached to the surface of the patterned area. (d) Zoomed-in SEM image to visibly realize the NPL attachment in the patterned area. Reprinted with permission from [52], copyright 2019, Royal Society of Chemistry.

eventually enhances the gain performance as a result of the reduction in the scattering rate. Due to the efficient exciton funneling from the CdS crown and reduction in scattering losses, ultralow optical gain thresholds were achieved for multicrown type-II NPLs.

\section{DOPING OF COLLOIDAL CDSE NPLS}

\section{A. Doping of CdSe NPLs by the High-Temperature-Based Nucleation-Doping Method}

Doping of CQDs has attracted much attention in the community because of its widespread utilization for bulk semiconductors. However, controllably doping CQDs and completely understanding the doping-induced special properties of CQDs are challenging. Moreover, the selfpurification of dopant ions in quantum-confined nanostructures and the nonuniform distribution of dopant ions among QD ensembles make these materials immature for practical utilization. In the last two decades, different colloidal methods based on an understanding of the precursor chemistry have helped successfully dope transition metal ions into CQDs. Several reports on the possible applications of these doped CQDs in different optoelectronic devices have been presented. Among them, Mnand Cu-doped CdSe, ZnS, ZnCdS, and ZnInS CQDs have been of major interest [4], [76]-[84]. These dopant ions create additional energy levels in the bandgap of the host semiconductor CQDs, which often result in a Stokes-shifted emission. These doped semiconductor CQDs have been used for different color conversion applications. The most widely studied dopant system, e.g., Mn-doped CQDs, emits a fixed orange emission, which originates from the $\mathrm{d}-\mathrm{d}$ transitions of Mn states [77]. This broad dopant-induced emission appears only if both the ${ }^{4} \mathrm{~T}_{1}$ and ${ }^{6} \mathrm{~A}_{1}$ states of the Mn dopant ions lie within the bandgap of the host semiconductor. The transfer of both photoexcited electrons and holes from the host semiconductor NC to dopant states results in a fixed orange emission from Mn-doped NCs. Therefore, a shift in the bandgap or alloying of the NCs does not result in tuning of the Mn dopant emission. In contrast, $\mathrm{Cu}^{+}$and $\mathrm{Ag}^{+}$doping of semiconductor host
CQDs results in tunable dopant-induced emission. $\mathrm{Cu}^{+}$and $\mathrm{Ag}^{+}$dopant ions typically possess a d-orbital state near the valence band, which can strongly capture the photoexcited holes [85], [86]. Therefore, the recombination of delocalized conduction band (CB) electrons with strongly localized holes in Cu-/Ag-doped CQDs results in a broad and Stokes-shifted dopant-induced emission. The tunability of the dopant-induced emission for $\mathrm{Cu}-/ \mathrm{Ag}$-doped CQDs is realized by changing the NC particle size or by alloying the semiconductor NC [86], [87]. Both the techniques result in a shift of the $\mathrm{CB}$ level, which in turn tunes the emission spectrum. However, the typical PL QYs for $\mathrm{Cu}-/ \mathrm{Ag}$-doped CdSe NCs are in the range of $10 \%-40 \%$ [88]-[90]. Furthermore, CdS shell deposition on CdSe cores has been observed to decrease the dopant-induced emission and hence the PL QYs [88].

Recently, in 2017, we reported successful doping of CdSe NPLs (possessing 3- and 4-ML thicknesses) using a high-temperature nucleation-doping method [20]. Briefly, the dopant precursor was introduced into the reaction just before the transformation of magic-sized CdSe clusters to platelets. For the synthesis of 4-ML undoped CdSe NPLs, cadmium acetate was added when the magic-sized clusters reached dimensions corresponding to $1.2 \mathrm{~nm}$. In the synthesis of Cu-doped NPLs, the optimized dopant precursor was added from $190{ }^{\circ} \mathrm{C}$ to $195{ }^{\circ} \mathrm{C}$, followed by a quick addition of cadmium acetate powder. The reactivity of the dopant precursor was adjusted by the addition of TOP. The reactivity of the dopant precursor was found to be imperative to achieving a pure population of MLs of desired thickness along with highly efficient dopant emission. The successful doping of these flat NCs was shown by using different experimental techniques, e.g., XPS, ICP:MS, and HAADF:EDS. Fig. 4(a)-(d) shows the HAADF:EDS images of Cu-doped CdSe NPLs. The presence of copper dopant ions along with host cadmium and selenium clearly supports the uniform distribution of dopant ions throughout the entire NPL. Fig. 4(e) shows the UV-vis absorption and PL emission spectra of Cu-doped 3- and-4 ML CdSe NPLs. Based on the absorption spectra, both the electron-heavy hole (e-hh) and electron-light hole (e-lh) transitions are clearly not affected by copper doping of CdSe host NPLs. Furthermore, the PL emission 

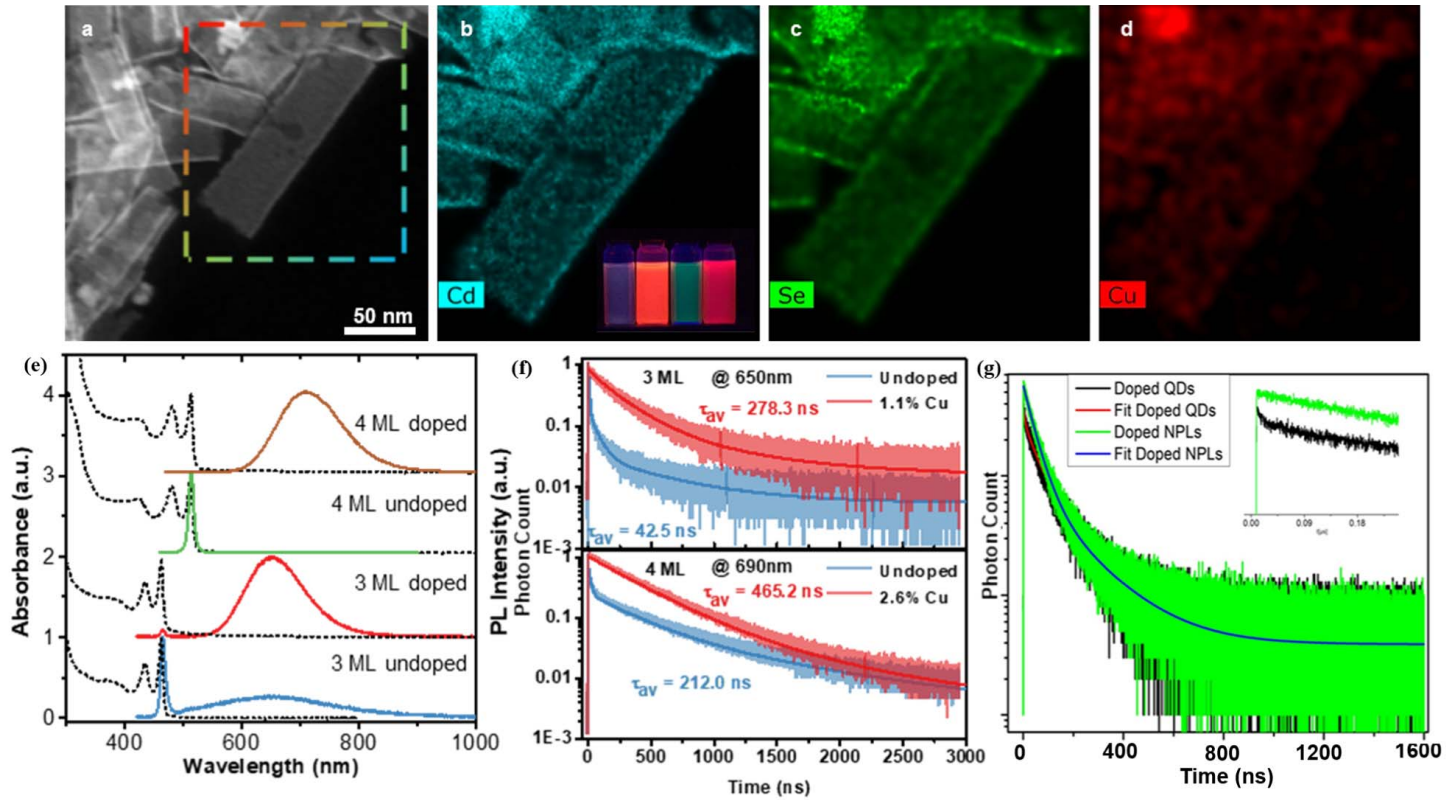

Fig. 4. (a)-(d) HAADF-STEM image and energy-dispersive X-ray maps of cadmium, selenium, and copper elements for 1.1\% Cu-doped 3-ML CdSe NPLs. (e) UV-vis absorption and PL spectra of 3- and 4-ML Cu-doped and undoped CdSe NPLs. TRF decay curves for (f) undoped and Cu-doped 3- and 4-ML CdSe NPLs recorded at the corresponding defect and dopant emission wavelengths, respectively, and (g) Cu-doped CdSe CQDs and NPLs. The inset of (b) shows the digital images of the undoped and Cu-doped CdSe 3-and 4-ML NPLs illuminated by a 365-nm UV lamp. Reprinted (adapted) with permission from [20] [(a)-(g) copyright 2017, Wiley].

spectra for doped samples show the appearance of a broad, dominant and Stokes-shifted emission along with a weak excitonic emission. Similar to CQDs, doping of copper into 3-4-ML CdSe NPLs leads to a broad and Stokes-shifted emission. The inset of Fig. 4(b) shows the digital images of undoped and doped NPLs under UV lamp excitation conditions. Interestingly, these Cu-doped CdSe NPLs possess very high PL QYs (70\%-90\%). This high PL QY for doped NPLs can be explained based on the time-resolved fluorescence (TRF) spectroscopy results. Fig. 4(g) shows the TRF decay curves for dopant emission from 3- to 4-ML NPLs compared with those for defect emission from undoped 3-4-ML CdSe NPLs. Clearly, the doped NPL emission shows a longer lifetime compared with the defect emission. Furthermore, a lifetime analysis suggests that the dopant-related emission in NPLs does not contain any fast decay channel ( $\sim 20 \mathrm{~ns}$ ) compared with similar Cu-doped CQDs, which supports the high PL QY in doped NPLs [see Fig. 4(h)] [20].

\section{B. Doping of CdSe NPLs by the Partial Cation Exchange Method}

In addition to the abovementioned high-temperature hot-injection method, in 2018, we reported the controlled synthesis of Cu-doped CdSe NPLs by a slow partial CE method [21]. Compared to the high-temperature nucleation-doping method, partial CE provides more flexibility for the introduction of dopant ions into host flat NPLs. With the tuning of the reactivity of the dopant precursor by using acetate- and nitrate-based salts for copper dopant ions and varying the TOP amounts, the doping mechanism and the induced properties were understood [21]. Fig. 5(a) shows a sketch of the partial CE reaction on CdSe NPLs. The application of slow partial CE using copper acetate allows full copper emission to appear on the order of days instead of over several minutes (e.g., by using reactive copper nitrate or the previously discussed high-temperature method). Fig. 5(b)-(d) shows the selected UV-vis absorption, PL emission, and TRF decay profiles for $\mathrm{Cu}$ doping using the slow partial CE method. As depicted, with increasing CE reaction time, the dopant emission intensity increases, with a considerable decrease in the excitonic emission. Lifetime analysis shows the appearance of a Cu-related decay channel consistent with the increase in the dopant emission. As discussed earlier, the emission mechanism of Cu-doped NPLs is similar to that of $\mathrm{Cu}$-doped CQDs. Basically, $\mathrm{Cu}$ states capture the photoexcited holes in $\mathrm{CdSe}$ that in turn radiatively recombine with delocalized CB electrons. The only striking difference is the large lateral area of the NPLs, which requires additional experimental procedures for converting undoped and partially doped subpopulations to fully and uniformly doped NPLs. This process was successfully understood and achieved by carefully controlling the partial CE reaction and annealing nonuniform-doped CdSe NPLs [21].

Apart from studies to obtain an understanding of the unique optical and electronic properties of these doped NCs, some interesting reports have studied dopant-induced 


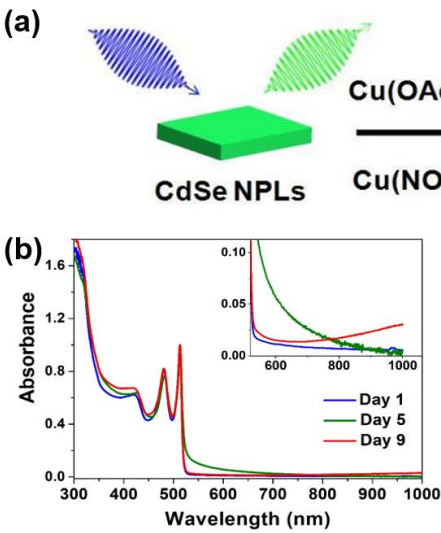

(e)

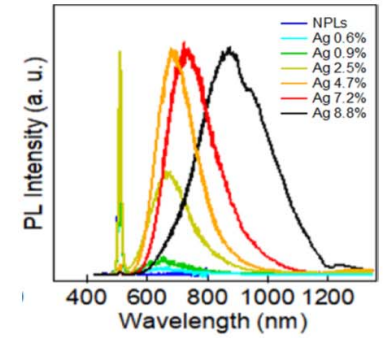

(f)

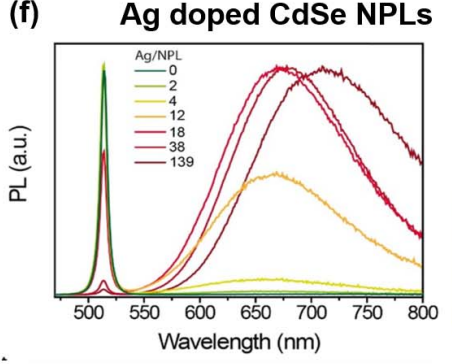

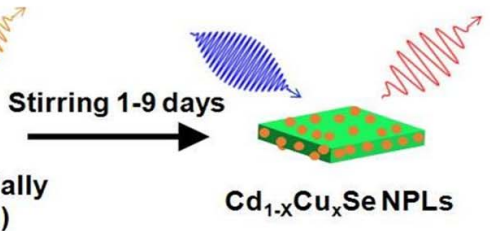

(c)
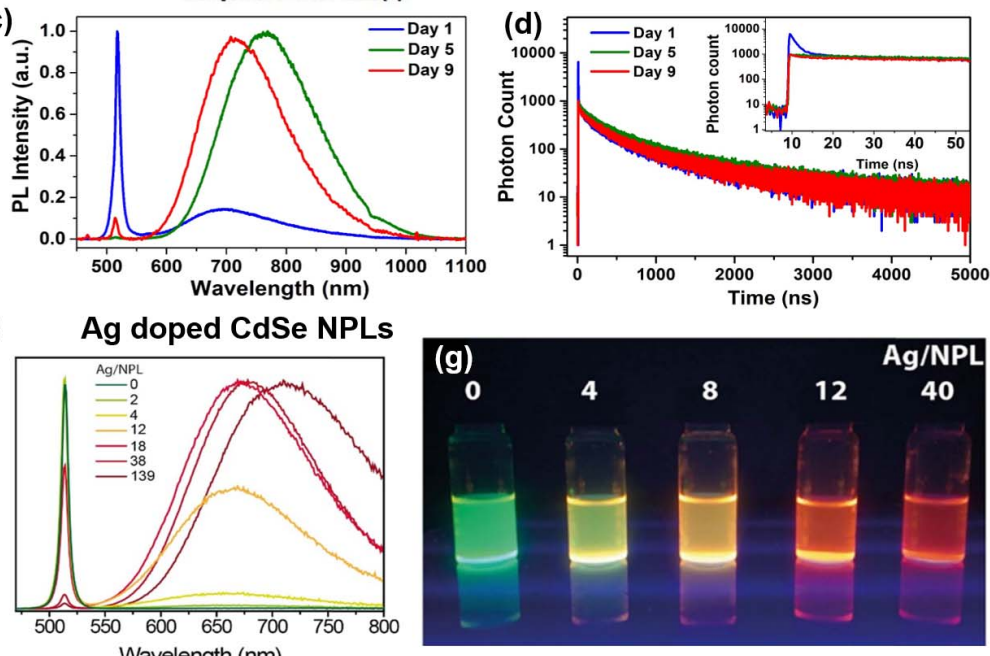

Fig. 5. (a) Copper doping procedure through the partial CE reaction using copper acetate and copper nitrate precursors. (b)-(d) Doping mechanism of steady-state UV-visible absorption, emission, and TRF decay curves for Cu-doped CdSe NPLs on different days of partial CE synthesis. (e)-(f) Silver doping of CdSe NPLs. Corresponding PL spectra of CdSe NPLs for various amounts of the Ag+ dopant. (g) Digital image of Ag+-doped CdSe NPL solutions with various doping levels. Reprinted (adapted) with permission from [21] [(a)-(d) copyright 2018, American Chemical Society], [23] [(e) copyright 2019, American Chemical Society), and [24] [(f) and (g) copyright 2019, American Chemical Society).

orbital hybridization of host CdSe orbitals. With the aim of controlling individual $\mathrm{CB} / \mathrm{VB}$ levels with respect to the cathode/anode, Wright and Meulenberg [87] and Wright et al. [91] showed that an increase in Cu(I) substitutional doping of host CdSe CQDs affects the lowest CB level in a way inconsistent with a quantum confinement theory. An increase in the Cu(I) doping levels in the host CdSe results in a decrease in the CB edge (only). This phenomenon has been shown by different experimental (X-ray absorption and ultraviolet photoemission spectroscopy) and theoretical methods [91]. Briefly, the PL emission consistently redshifts with an increase in the copper level. The energy position of the $\mathrm{Cu} 4 \mathrm{~s}$ orbitals is close to that of the host Cd 5 s orbital, which results in orbital hybridization. However, the particle size distribution-related shift of the dopant emission in a doped NC ensemble could be confused with this observation. Here, the carefully controlled $\mathrm{Cu}$ levels in atomically flat NPLs, where the strong quantum confinement in 1-D eliminates the possibility of a size distribution-related redshift of the emission, help uniquely reveal the dopant-induced orbital hybridization in Cu-doped CdSe NPLs.

Very recently, by utilizing a similar partial CE method, Ag doping of CdSe NPLs was also shown [23], [24]. Briefly, as shown in Fig. 5(e) and (f), by varying the Ag dopant levels in the host CdSe NPLs, the dopant-to-excitonic emission ratio can be tuned, which results in a color change in the emission from green to red. Furthermore, at high $\mathrm{Ag}$ levels, such as $8.8 \%$, with respect to Cd levels, the emission spectrum can be tuned up to $870 \mathrm{~nm}$ with considerable PL QYs of 45\%-55\%. Along with cationic doping in host CdSe NPLs, anionic doping as Te element was also shown by Dubertret group in 2015 [92]. The authors added different concentrations of tellurium (Te) precursors along with selenium (Se) to achieve low doped (CdSe:Te) to alloyed $\mathrm{CdSe}_{\mathrm{x}} \mathrm{Te}_{(1-x)}$ NPLs. Similar to $\mathrm{Cu}$ and $\mathrm{Ag}$ cationic dopants, at low doping concentrations, Te ions also act as deep hole traps, which result in a broad Stokes-shifted emission. For Te ions (>30\%), the $\mathrm{CdSe}_{x} \mathrm{Te}_{(1-x)}$ NPLs display emission behavior of an alloyed material with a bowing of the bandgap.

\section{AP PLICATION S OF COLLOIDAL CDX ( $\mathrm{X}=\mathrm{S}, \mathrm{SE})-\mathrm{BASED}$ NPLS}

\section{A. Light-Emitting Devices}

QD-based LEDs (QD-LEDs) are regarded as the nextgeneration display technology. With recent advancements in the synthesis of reproducible near-unity efficiency CQDs and improved device engineering of different device layers, QD-LEDs have reached their maximum performance limits [93], [94]. Benefiting from the ideal combination of high brightness (up to $\sim 200000 \mathrm{~cd} \mathrm{~m}^{-2}$ ), low operating voltage ( $V$ turn-on $<2 \mathrm{~V}$ ), outstanding color purity 
(FWHM $\sim 30 \mathrm{~nm}$ ), high efficiency with a long lifetime, flexibility, and low processing cost, QD-LEDs have been extensively studied in the last two decades. [95], [96]. In a short span, QD-LEDs have entered display markets after the first demonstration of a CQD-LED, dated to 1994, in the research laboratories of Prof. Alivisatos at the University of California [97]. By understanding organic light-emitting devices (OLEDs) and polymer light-emitting devices (PLEDs), the development of CQD-LEDs has gained advantages and has been accelerated. The recent addition of CQWs to the colloidal NC family, possessing astonishingly good optical properties due to the precise control of thickness in the vertical direction, shows great potential to further improve QD-LEDs. However, to date, only limited reports on CQW-based LEDs are available [28], [67], [71], [98]. The goal of this section is to discuss the recent work in the last five years that involves successful utilization of CdSe-based CQWs in hybrid organic-inorganic LEDs. Finally, we will discuss some of the current directions for CdSe-based CQW-LEDs, concentrating on the realization of next-generation displays and high-quality lighting with higher efficiency and superior color gamut. For a detailed understanding of the QD-LED device operation and the evolution of the improvements over the last two decades, readers are referred to some specific detailed reviews [63], [93]. The important performance metric, namely, the external quantum efficiency (EQE), is defined as the product of the internal quantum efficiency (IQE) and the light out-coupling efficiency $\left(\eta_{\text {out }}\right)$. However, the maximum value of $\eta_{\text {out }}$ is limited to only $\sim 20 \%$ for typical LEDs fabricated on indium tin oxide (ITO)-coated glass substrates [99], [100]. Thus, nearly $80 \%$ of the generated light is trapped in the transparent conducting oxide layer or substrate layers due to the total internal reflection caused by the refractive index mismatch between the different LED layers and air. The typical EQE expression used in LED performance evaluation is $\mathrm{EQE}=\eta_{\text {out. }}$.r.q. $\gamma$, where $\eta_{\text {out }}$ is the light out-coupling efficiency as described earlier, $r$ is the fraction of excitons that radiatively recombine, $q$ is the PL QY of the emissive layers, and $\gamma$ is the charge balance. The product of r.q. $\gamma$ is typically called the IQE of LEDs [101].

CdSe-based semiconductor NPLs uniquely combine the handing advantages of solution-based materials similar to CQDs with the physical properties of 2-D QWs synthesized by metal organic chemical vapor deposition (MOCVD) and molecular beam epitaxy (MBE). Solution-processed 2-D semiconductor CdSe-based NPLs are projected to be ideal emissive materials for nextgeneration LEDs. Fundamentally, NPL-based electroluminescent devices exhibit some advantages over QD-based electroluminescent devices, including narrow electroluminescence (EL) emission linewidths resulting from the highly homogeneous platelet thicknesses, tunable emission in the visible region induced by precise control of the thickness at the atomic level, high PL QYs, strong exciton and biexciton binding energies, and suppressed Auger recombination [26]. The first report on a colloidal
CdSe NPL-based LED appeared in 2014 [28]. Core-shell CdSe/CdZnS NPLs were used as an emitting layer in the hybrid structure and showed an EQE of $0.63 \%$, with a luminance of $4499 \mathrm{~cd} / \mathrm{m}^{2}$. The FWHM of emission was narrow and varied from 25 to $30 \mathrm{~nm}$. In the following two years, pure green EL emission from CdSe and CdSeS NPLs with core and core-crown architectures was demonstrated [67], [102], [103]. Interestingly, the green EL emission from these materials shows a very narrow FWHM of $12-14 \mathrm{~nm}$, which is the narrowest emission shown for any colloidally synthesized material. The core-crown architecture also leads to a lower turn-on voltage and a higher EQE of 5.0\% [67]. Fig. 6(a)-(d) shows the LED devices made of CdSe/CdS NPLs, showing the EL spectra at different operating voltages, Commision International de l'Eclairage (CIE) color coordinates for green EL emission, a histogram of the EQE values for different sets of devices, and an EL photograph under an applied voltage of $6 \mathrm{~V}$.

Recently, core-shell CdSe/CdZnS NPLs have been shown to possess improved EQEs of up to $5.73 \%$ with the narrow EL linewidths of $25 \mathrm{~nm}$ and a luminance of $1540 \mathrm{~cd} / \mathrm{m}^{2}$ [98]. These results have been achieved by using conjugated polymers with polar and electrolytic side chains [conjugated polyelectrolytes (CPEs)] as the electron transport layer (ETL), also called P1 ETL. Fig. 6(e)-(h) shows the device characteristics of red-emitting core-shell NPLs. Fig. 6(e) shows the EL spectra of the red-emitting NPL-LED with P1 ETL under increasing driving voltage from 4 to $11 \mathrm{~V}$. In the EL spectra, no contribution from the polymer emitting layers is observed even at the higher operating voltage of $11 \mathrm{~V}$. The color purity of these core-shell NPLs is shown by the CIE color coordinates of $x=0.71$ and $y=0.29$, which corresponds to $98 \%$ saturated deep-red light ideal for wide-gamut RGB displays. Further improvements in the performance of these devices were achieved by exposing them to humid air, which strongly enhanced the performance of these devices and led to the EQE of $8.39 \%$, a record in the CQW category [see Fig. 6(g)] [98]. Even without encapsulation, these devices were reported to be stable under continuous operation in air for many hours [see Fig. 6(h)].

Recently, core-crown NPLs having type-II band alignment and Stokes-shifted emission with very high PL QYs ( $85 \%)$ were also tested for their performance in LED devices [71]. Interestingly, these materials show yellow-orange EL emission with a very high luminance of $34520 \mathrm{~cd} / \mathrm{m}^{2}$, along with a low operating voltage of $1.9 \mathrm{~V}$ and a reasonably high EQE of $3.57 \%$. Furthermore, CdSe NPLs possessing 3-ML vertical thickness and emitting in the blue region $(\sim 460 \mathrm{~nm})$ are available and shown to be useful as the blue emitter in lasing studies [38]. However, there is no report on LED devices with these blue-emitting NPLs. These blue-emitting 3-ML CdSe NPLs, however, exhibit poor PL QYs currently compared with similar thicker NPLs. Moreover, these NPLs tend to form rolls because of very small thickness and large lateral dimensions that can affect their device fabrication. Fan et al. [102] reported the tuning of the EL from 520 to $490 \mathrm{~nm}$ 

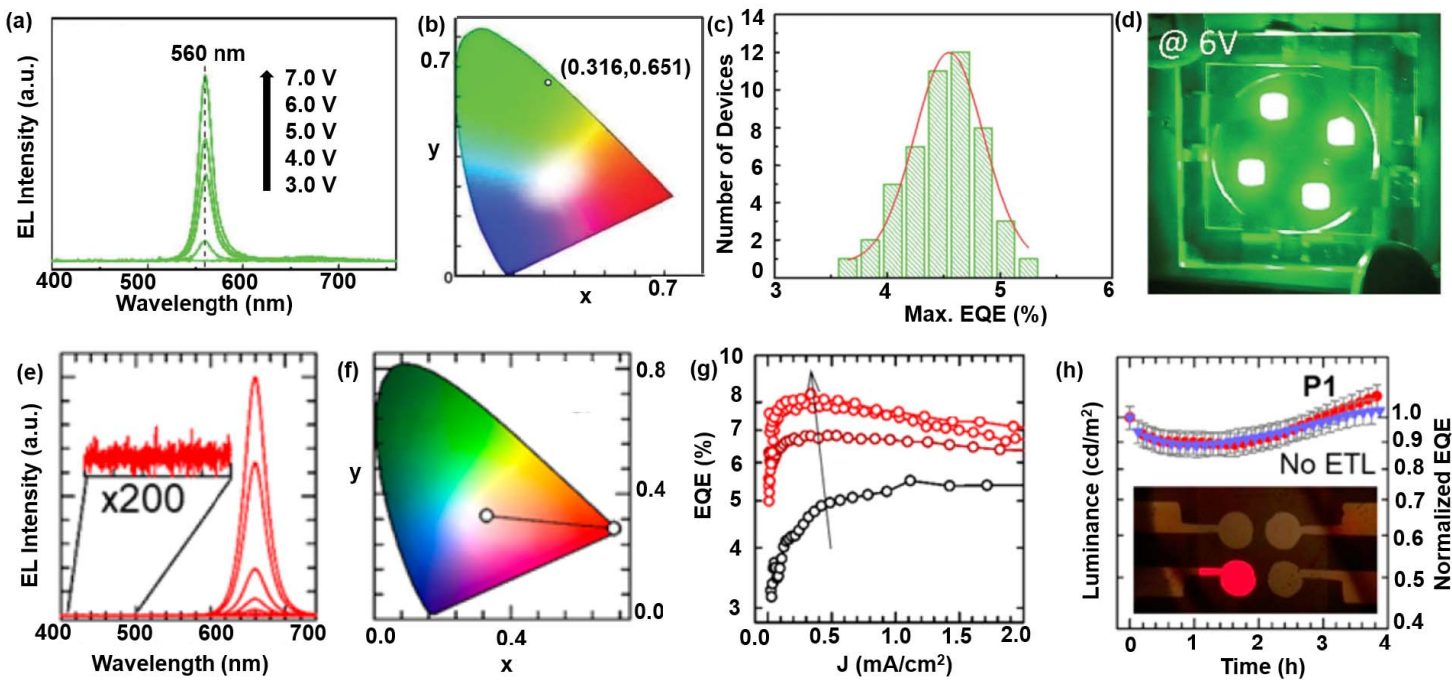

Fig. 6. (a) Evolution of the EL spectra of a green 5-ML CdSe NPL-LED with increasing driving voltage. (b) CIE color coordinates of (0.316, 0.651 ) corresponding to the green emission spectra in (a). (c) Histogram of maximum EQE (max. $\eta_{E Q E}$ ) measured for 50 devices. (d) EL photograph of a green LED under an applied voltage of 6 V. (e) EL spectra of the red-emitting CdSe/CdZnS NPL-LED with PI ETL under increasing driving voltage from 4 to $11 \mathrm{~V}$. Inset: enlarged EL at $11 \mathrm{~V}$ between 400 and 500 nm, which highlights the absence of EL contributions from the polymeric interlayers. (f) CIE color coordinates for the EL reported in (e). (g) EQE for the NPL-LED with P1 ETL as a function of $J$ which is recorded for the pristine device in a nitrogen atmosphere (black circles) and after exposure to atmospheric conditions for 15, 30, and 330 min. (h) Normalized EQE for red-emitting CdSe/CdZnS NPL-LEDs with and without P1 ETL are shown by red and blue dots, respectively. This is monitored over $4 \mathrm{~h}$ of continuous operation in humid (50\%) air. The error bars are shown in gray. The LEDs were stored in air for $5 \mathrm{~h}$ (prior to the measurement) to saturate the efficiency enhancement effect shown in (e). Inset: working LED photograph with a $P 1$ interlayer and an Al cathode. Under a driving bias of 6 V, bright emission over the entire device area of 5.4 mm ${ }^{2}$ is observed. Reprinted (adapted) with permission from [67] [(a)-(d) copyright 2016, Royal Society of Chemistry] and [98] [(e)-(h) copyright 2018, American Chemical Society].

by alloying of 4-ML CdSe ${ }_{1-x} S_{x}$ NPLs. However, in order to achieve blue-emitting LEDs from CdSe-based NPLs, further optimization is needed for 3-ML CdSe NPL-based materials and their device fabrication processes.

Although these NPL-based devices do not show better device performance than the best-reported QD-LEDs, these devices have the potential for a wide scope of improvement. All studied NPL-based LEDs along with two similar QD-LEDs are summarized in Table 1. One possible reason for the lower performance might be the very limited exploration of LED devices with NPL-based emissive layers. Compared to QD materials and related devices, these NPLs have recently been introduced into the colloidal NC family. The PL QYs of giant core-shell CQDs have reached near unity, whereas typical NPLs used in LED devices possess lower PL QYs and poor stability. In the last few years, new improvements in achieving near-unity and very stable core-shell NPLs have been reported [52], [58]. Compared to the earlier reported synthesis methods that used the room-temperature-based c-ALD procedure for growing shell materials on core NPLs, new improved synthesis methods using high-temperature hot-injection growth procedures showed greatly improved PL QYs and stability for CdSe/ZnS NPLs [58]. These stable and near-unity efficiency NPL materials would enable the NPL-based devices to reach the performance of QD-LEDs. Furthermore, as recently reported, the effective use of the ETL improves the performance of moderately efficient
NPLs from $0.63 \%$ to $8.39 \%$. Further improvements in charge transport layers (CTLs) are expected to improve the performance of the narrow-emitting NPLs. To date, no reports on the use of all-inorganic NPL-based LEDs have been presented. By utilizing the experience gained in using inorganic CTLs for QD-LEDs, similar improvements are expected for NPL-based LEDs in the near future. Recently, we showed that flat 2-D NPLs can be deposited on glass substrates as a controlled assembly (e.g., lying flat on the substrates) [52]. These 2-D NPLs possess highly anisotropic emission, which is directed perpendicular to the heavy hole exciton transition dipole distribution along the plane of the flat NPLs. However, in spin-coated films, these NPLs are randomly distributed and sometimes form long stacks, which results in lower emission efficiencies and moderately directed emission. With surface modification using a linker molecule, these 2-D NPLs can be horizontally deposited on substrates, which can enhance their directed emission. Unlike CQDs, which show isotropic emission, this highly directed emission property in colloidal NPLs, if used properly in a device configuration, can increase the out-coupling efficiency of LEDs in displays and lighting [52].

\section{B. ASE and lasing}

NPLs possess excellent optical and electronic properties, such as ultranarrow spontaneous emission as a result 
Table 1 Comparison of Different LEDs Based on CQWs as the Emissive Layer With Similar Selected CQD LEDs

\begin{tabular}{|c|c|c|c|c|c|c|c|}
\hline Devices & $\begin{array}{l}\lambda_{\max } \\
(\mathbf{n m})\end{array}$ & $\begin{array}{l}\text { FWH } \\
\text { M of } \\
\text { EL } \\
(\mathbf{n m})\end{array}$ & $\begin{array}{l}\text { Turn-on } \\
\text { voltage } \\
\left(V_{\text {on }}, V\right)\end{array}$ & $\begin{array}{l}\mathbf{L}_{\max } \\
\left(\mathbf{c d ~ m ^ { - 2 }}\right)\end{array}$ & $\begin{array}{l}\text { EQE }_{\max } \\
(\%)\end{array}$ & Remarks & $\begin{array}{c}\text { Refere } \\
\text { nce }\end{array}$ \\
\hline CdSe NPLs & 515 & - & 5.5 & - & - & $\begin{array}{c}\text { Architecture- } \\
\text { ITO/PEDOT:PSS/TPD/NPLs/Alq } \\
\text { 3/Al }\end{array}$ & [103] \\
\hline $\begin{array}{l}\mathrm{CdSe}_{\mathrm{x}} \mathrm{S}_{1-\mathrm{x}} \\
\mathrm{NPLs}\end{array}$ & 520 & 12.5 & 2.1 & $\sim 90$ & - & $\begin{array}{l}\text { Core-only NPLs with alloying of } \\
\text { the core with sulfur }\end{array}$ & [102] \\
\hline $\begin{array}{l}\text { CdSe-CdS } \\
\text { core-crown } \\
\text { NPLs }\end{array}$ & 515 & 14 & 2.2 & 33,000 & 5.0 & $\begin{array}{c}\text { Architecture: } \\
\text { ITO/PEDOT:PSS/TFB/NPL/ZnO/ } \\
\text { Al }\end{array}$ & [67] \\
\hline $\begin{array}{l}\mathrm{CdSe} / \mathrm{CdTe}_{\mathrm{x}} \\
\mathrm{Se}_{1-\mathrm{x}} \text { core- } \\
\text { crown NPLs }\end{array}$ & 599 & -- & 1.9 & 34,520 & 3.57 & $\begin{array}{l}\text { Type-II NPL with a Stokes-shifted } \\
\text { emission }\end{array}$ & [71] \\
\hline $\begin{array}{l}\mathrm{CdSe} / \mathrm{CdZn} \\
\mathrm{S} \text { core-shell } \\
\text { NPLs }\end{array}$ & 645 & $25-30$ & 4.7 & 4499 & 0.63 & $\begin{array}{c}\text { Architecture: } \\
\text { TO/PEDOT:PSS/PVK/NPL- } \\
\text { MPA/ZnO/Al }\end{array}$ & {$[28]$} \\
\hline $\begin{array}{l}\mathrm{CdSe} / \mathrm{CdZn} \\
\mathrm{S} \text { core-shell } \\
\text { NPLs }\end{array}$ & 658 & 25 & 4.0 & - & $3.76-8.39$ & $\begin{array}{c}\text { Functionalized copolymer (P1) } \\
\text { used as the ETL, } \\
\text { architecture: } \\
\text { ITO/PEDOT:PSS/PVK/NPL/x/y } \\
\text { (x/y: P1/Al, -/Al, P1/Ba and Al, } \\
\mathrm{Ba} / \mathrm{Al}, \mathrm{P} 1 / \mathrm{Ag},-/ \mathrm{Ag})\end{array}$ & [98] \\
\hline $\begin{array}{l}\mathrm{CdSe} / \mathrm{CdS} \\
\text { core-shell } \\
\text { CQDs }\end{array}$ & 610 & 30 & - & 6,000 & 2.12 & $\begin{array}{c}\text { Architecture: } \\
\text { ITO/PEDOT:PSS/BiVB- } \\
\text { MeTPD/QD/TPBI/CsF/Al }\end{array}$ & {$[10]$} \\
\hline $\begin{array}{l}\mathrm{CdSe} / \mathrm{ZnS} \\
\mathrm{CQDs}\end{array}$ & $\begin{array}{c}460- \\
650\end{array}$ & $30-40$ & - & - & $0.2-2.7$ & $\begin{array}{c}\text { Architecture: } \\
\text { ITO/PEDOT:PSS/spiroTPD/QD/T } \\
\text { PBI/Mg:Ag/Ag }\end{array}$ & [104] \\
\hline
\end{tabular}

of the absence of nonhomogenous broadening and giant oscillator strength leading large stimulated emission cross section, which makes them highly favorable candidates for lasing and optical gain applications [105]. In addition, NPLs with their 2-D electronic profiles possess a high excitonic density of states beneficial for stimulated emission, which is important for obtaining high modal gain. Due to their 2-D electronic structure, several excitons in NPLs can occupy the same energy level, providing a solid platform for optical gain, unlike CQDs, which can only support two band-edge excitons. In addition, in NPLs, the Auger recombination rate is likely reduced compared to 0-D CQDs due to the stricter momentum conservation as a result of quantization in only one direction. Furthermore, the high energy carriers in NPLs relax to the band edge faster than the recombination time, which is advantageous for lasing applications [106].

Using core NPLs and different NPL heterostructures, optical gain over the entire visible spectrum was recently demonstrated. Blue, green, and yellow ASEs were obtained with low thresholds using core NPLs with different thicknesses, while red ASE was achieved using CdSe/CdS core/shell NPLs, as presented in Fig. 7(a) and (b) [38]. For the green region, the reported ASE thresholds are $\sim 6-15$ and $\sim 40 \mu \mathrm{J} / \mathrm{cm}^{2}$ for core and core/crown NPLs, respectively [38], [68], [105]. One would expect a lower threshold for core/crown NPLs due to their enhanced absorption cross section and better periphery passivation. This unexpected result can be attributed to the difference in the film formation leading to different loss coefficients 

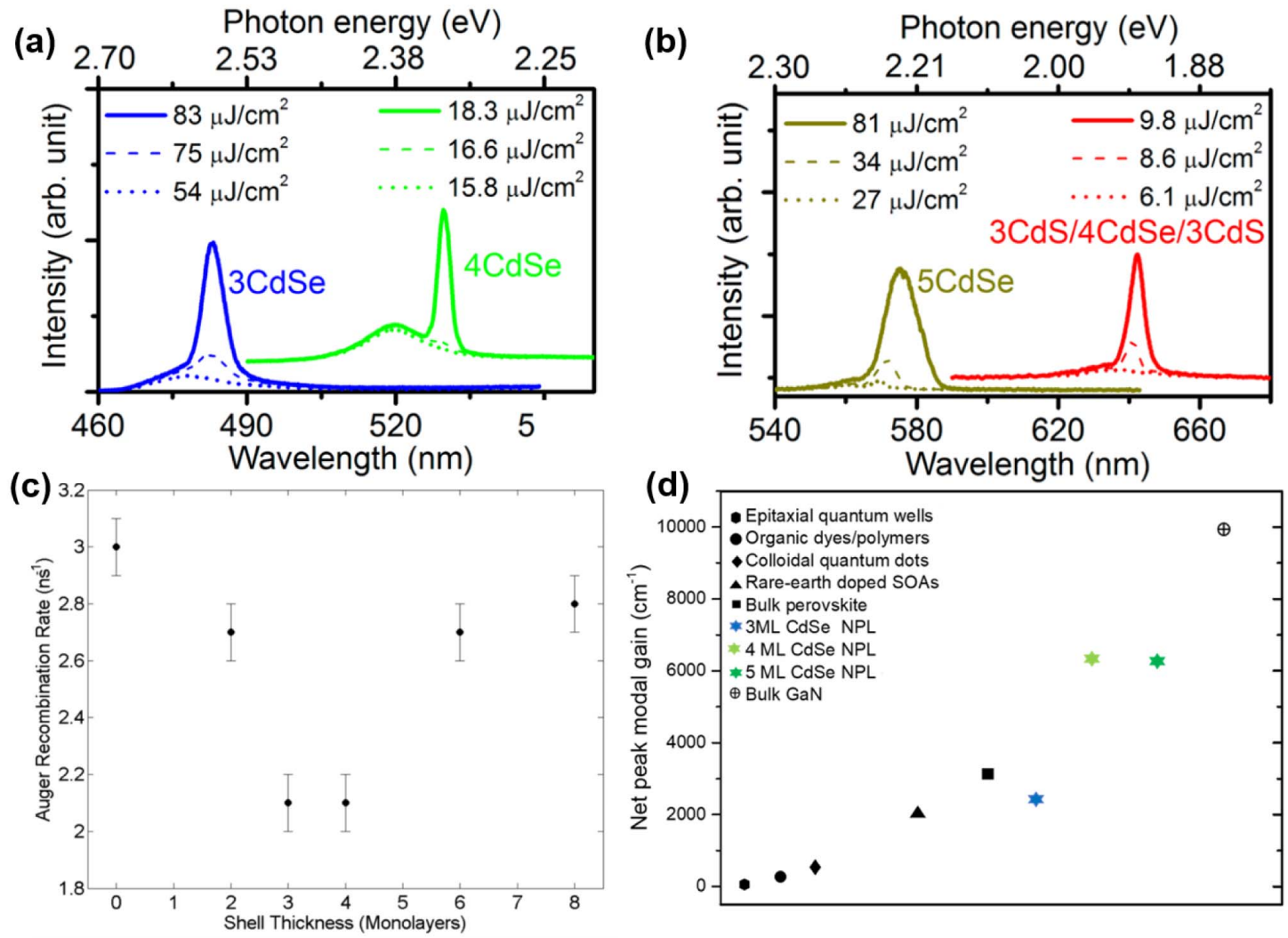

Fig. 7. (a) and (b) ASE spectra of CdSe NPLs with 3-5-ML vertical thickness and core-shell NPLs with a 3-ML CdS shell. (c) Auger recombination rate, $A$, as defined in the text, as a function of shell thickness for CdSe/CdS core/shell NPLs with the core thicknesses of 4 MLs [110]. (d) Peak net modal gain coeficients of CdSe NPLs with different vertical thicknesses and lateral areas. The peak net modal gains reported for other systems are also presented: epitaxial QWs [115], organic dyes/polymers [116], CQDs [117], [118], rare-earth-doped SOAs [119], bulk perovskites [120], 3-5-ML CdSe NPLs [111], and bulk GaN [121]. The empty symbol indicates a measurement at cryogenic temperature. Reprinted (adapted) with permission from [38] [(a) and (b) copyright 2014, American Chemical Society], [110] [(c) copyright 2017, American Chemical Society], and [111] [(d) copyright 2019, American Chemical Society].

and optical confinement factors. Also, here, it is worthwhile to note that although the comparison between the ASE thresholds is very useful for evaluating the materials' aptitude toward gain, it does not thoroughly describe the gain performance of the materials since the other parameters besides intrinsic gain characteristics of materials strongly affect the threshold, such as film formation and optical confinement factor. Employing alloyed $\mathrm{CdSe}_{x} \mathrm{~S}_{1-x}$ NPL heterostructures also leads to highly tunable low-threshold ASE in the green region [107]. The origin of the redshifted green ASE peak in these NPLs was attributed to the biexcitonic gain observed in type-I systems.

Realizing optical gain in the blue region is difficult since for blue emission, generally, smaller NCs that have faster Auger rates and a higher density of trap states as a result of higher surface-to-volume ratios are needed. The reported ASE thresholds for the blue region in NPLs are $\sim 50 \mu \mathrm{J} / \mathrm{cm}^{2}$ [38] and are comparable to or lower than the best results for CQDs [49], [108]. ASE was also obtained in the violet region by employing CdS/ZnS core/shell NPLs. These CdS/ZnS core/shell NPLs exhibit a reasonably low ASE threshold of $\sim 50 \mu \mathrm{J} / \mathrm{cm}^{2}$ that is similar to the thresholds in the blue region [109]. The ASE threshold for red-emitting core/shell NPLs was found to be as low as $6 \mu \mathrm{J} / \mathrm{cm}^{2}$ [38]. This exceptionally low threshold compared to CdSe/CdS CQDs is a result of the large absorption cross section and narrow emission linewidth. The Auger rate in these core/shell NPLs exhibits a nonmonotonic dependence on the shell thickness, as shown in Fig. 7(c) [110]. The Auger rate first decreases and then increases after reaching a minimum, which was attributed to the oscillations in the overlap between the final and initial electron wavefunctions with the change in the number of shell layers [110]. Such nonmonotonic behavior has not been observed in CQDs, possibly due to the inhomogeneous broadening, which is not present in NPLs. In the red region, type-II CdSe/CdSeTe NPLs also exhibit a low ASE of threshold $\sim 26 \mu \mathrm{J} / \mathrm{cm}^{2}$ with a high modal gain coefficient $\left(g_{\text {modal }} \approx\right.$ $930 \mathrm{~cm}^{-1}$ ) [72]. These results present more than an order of magnitude enhancement over the previous reports for type-II NCs [9], [62].

Recently, stable ASEs with ultralow thresholds were observed in the red region for advanced NPL 
Table 2 Comparison of ASE Thresholds of CQWs With Those of Selected CQDs in the Visible Region

\begin{tabular}{|c|c|c|c|c|}
\hline Material & $\begin{array}{l}\text { ASE } \\
\text { threshold }\end{array}$ & $\lambda(\mathrm{nm})$ & Remarks & Reference \\
\hline 3 ML CdSe NPLs & $50 \mu \mathrm{J} / \mathrm{cm}^{2}$ & $480 \mathrm{~nm}$ & Core-only NPLs & [38] \\
\hline $\mathrm{CdS} / \mathrm{CdZnS}$ core/shell NPLs & $75 \mu \mathrm{J} / \mathrm{cm}^{2}$ & $480 \mathrm{~nm}$ & $\begin{array}{l}\text { Synthesized by the c- } \\
\text { ALD technique }\end{array}$ & {$[109]$} \\
\hline $\mathrm{CdSe}_{\mathrm{x}} \mathrm{S}_{1-\mathrm{x}} \mathrm{NPLs}$ & $120 \mu \mathrm{J} / \mathrm{cm}^{2}$ & $500-540 \mathrm{~nm}$ & $\begin{array}{l}\text { Tunable ASE by } \\
\text { controlled alloying }\end{array}$ & [107] \\
\hline 4 ML CdSe NPLs & $6 \mu \mathrm{J} / \mathrm{cm}^{2}$ & $532 \mathrm{~nm}$ & Core-only NPLs & {$[105]$} \\
\hline $\begin{array}{l}\mathrm{CdSe} / \mathrm{CdSeTe} / \mathrm{CdS} \\
\text { core/multicrown NPLs }\end{array}$ & $4 \mu \mathrm{J} / \mathrm{cm}^{2}$ & $610 \mathrm{~nm}$ & Type-II NPLs & [75] \\
\hline $\begin{array}{l}\mathrm{CdSe} / \mathrm{CdSeTe} \text { core/crown } \\
\text { NPLs }\end{array}$ & $26 \mu \mathrm{J} / \mathrm{cm}^{2}$ & $615 \mathrm{~nm}$ & Type-II NPLs & {$[72]$} \\
\hline $\mathrm{CdSe} / \mathrm{CdZnS}$ core/shell NPLs & $7 \mu \mathrm{J} / \mathrm{cm}^{2}$ & $625 \mathrm{~nm}$ & $\begin{array}{l}\text { Shell synthesized at } \\
\text { high temperature }\end{array}$ & {$[58]$} \\
\hline $\mathrm{CdSe} / \mathrm{CdS}$ core/shell NPLs & $6 \mu \mathrm{J} / \mathrm{cm}^{2}$ & $640 \mathrm{~nm}$ & $\begin{array}{l}\text { Shell synthesized by } \\
\text { the c-ALD technique }\end{array}$ & [41] \\
\hline $\mathrm{CdZnS} / \mathrm{ZnS}$ core/shell CQDs & $60 \mu \mathrm{J} / \mathrm{cm}^{2}$ & $450 \mathrm{~nm}$ & $\begin{array}{l}\text { Gradient-alloyed core- } \\
\text { shell interface }\end{array}$ & [108] \\
\hline CdSe/ZnCdS core/shell CQDs & $150 \mu \mathrm{J} / \mathrm{cm}^{2}$ & $565 \mathrm{~nm}$ & - & {$[49]$} \\
\hline $\begin{array}{l}\mathrm{CdSe} / \mathrm{CdSe} \mathrm{S}_{1-\mathrm{x}} / \mathrm{CdS} \\
\text { core/alloyed shell CQDs }\end{array}$ & $6 \mu \mathrm{J} / \mathrm{cm}^{2}$ & $645 \mathrm{~nm}$ & $\begin{array}{l}\text { Gradient-alloyed shell } \\
\text { for smooth interface }\end{array}$ & {$[122]$} \\
\hline
\end{tabular}

heterostructures. CdSe/CdS@CdS core/crown@shell NPLs resembling a platelet in a box were shown to exhibit ultralow thresholds and stable ASEs over more than $6 \mathrm{~h}$ [16]. These core/crown/shell NPLs exhibited better ASE performance compared to their core, core/crown, and core/shell counterparts [16] as a result of their better surface passivation and quasitype-II structure with the reduced Auger rate because of the reduced overlap between the electron and hole wavefunctions. Additionally, by using type-II NPLs with a CdSe/CdSeTe/CdS multicrown architecture, an ultralow threshold of $\sim 4 \mu \mathrm{J} / \mathrm{cm}^{2}$ with a very low waveguide loss coefficient was demonstrated [75]. In these core/multicrown NPLs, scattering losses are reduced due to the CdS crown, which prevents the formation of stacking. To date, type-II NPLs have only been explored in terms of the core/crown geometry, where the interfacial area is small, and extending type-II NPLs to the core/shell geometry should further reduce their gain threshold by enhancing the charge transfer states and reducing the Auger rate [18]. These engineered core/crown@shell NPLs and core-multicrown NPLs exhibit excellent optical gain performance and enhanced stability, which are key factors in realizing colloidal lasers. Table 2 summarizes the ASE thresholds of the studied CQWs along with the selected CQDs in the visible region.

The modal gain coefficient quantifying the light amplification per unit length is another key figure of merit for lasing applications. In CQDs, modal gain coefficients are small and quickly saturate because CQDs cannot support multiple excitons $(>2)$ at the band edge and generally exhibit fast Auger rates [62], [63]. However, due to their 2-D electronic structure, NPLs can have a high population inversion density and multiple excitonic species available for gain. As a result, NPLs exhibit large modal gain coefficients. Large net modal gain coefficients on the order of $600 \mathrm{~cm}^{-1}$ were initially reported for type-I core/crown and core/shell NPLs [41], [68]. However, the net modal gain of NPLs has recently been reported to reach $6600 \mathrm{~cm}^{-1}$ by producing close-packed films of NPLs [111]. The material gain of these NPL thin films at room temperature was estimated to be $\sim 15000 \mathrm{~cm}^{-1}$, which is comparable to the modal gain of bulk GaN at cryogenic temperatures. As shown in Fig. 7(d), the net modal gain of NPLs significantly outperforms that of other colloidal systems, making NPLs suitable candidates for the next-generation lasing applications.

After the demonstration of ASE, lasing was demonstrated by integrating NPLs into optical cavities. Using core, core/crown, and core/shell NPLs, lasing was demonstrated over the entire visible spectrum using one-, two-, and three-photon excitations in Fabry-Peìrot cavities [38], [68], [112]. The first demonstration of lasing was achieved by using core/crown NPLs in a two-photon absorption pumped vertical cavity, which was realized by using all-solution-processed distributed Bragg reflectors consisting of alternately stacked $\mathrm{SiO}_{2}$ and $\mathrm{TiO}_{2}$ nanoparticles [68]. A schematic of this verticalcavity surface-emitting laser (VCSEL) is shown in Fig. 8(a), and Fig. 8(b) shows the emission spectra of the VCSEL. The lasing threshold of this proof-of-concept laser was only $2.49 \mathrm{~mJ} / \mathrm{cm}^{2}$ under 2-PA pumping, as shown in Fig. 8(c). Later, lasing under continuous-wave (CW) 1-PA excitation was realized with solution-processed materials for the first time using similar Bragg reflectors, which is a key step toward realizing next-generation 
(a)

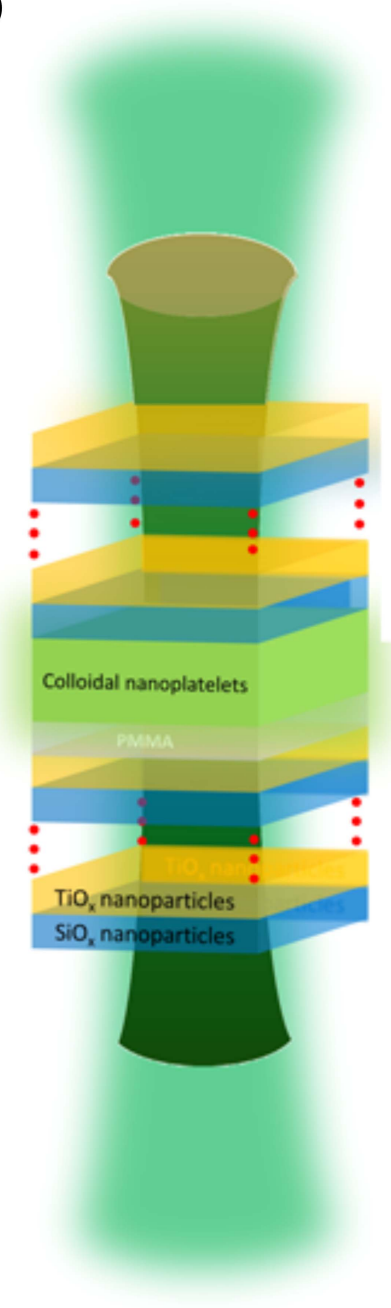

(b)

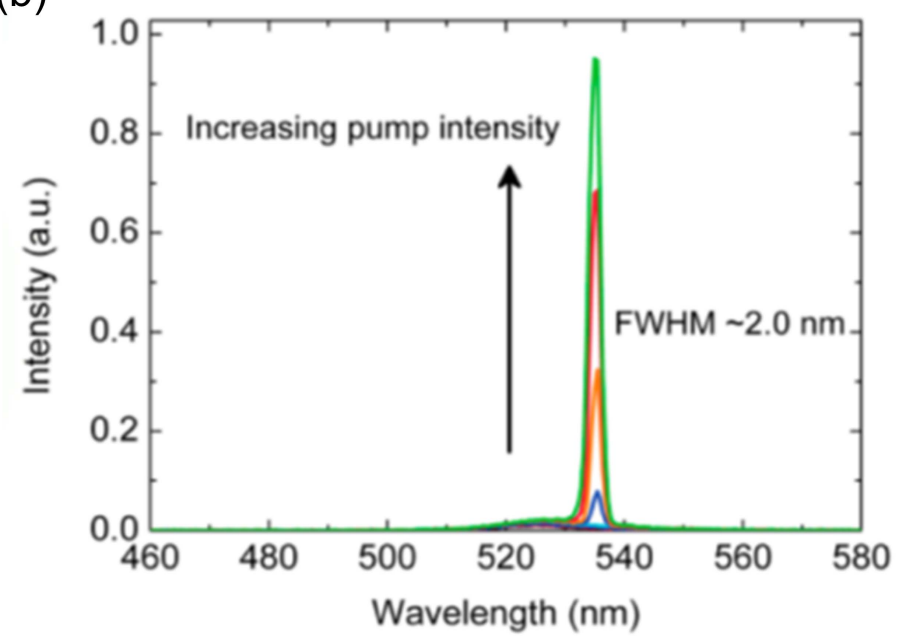

(c)

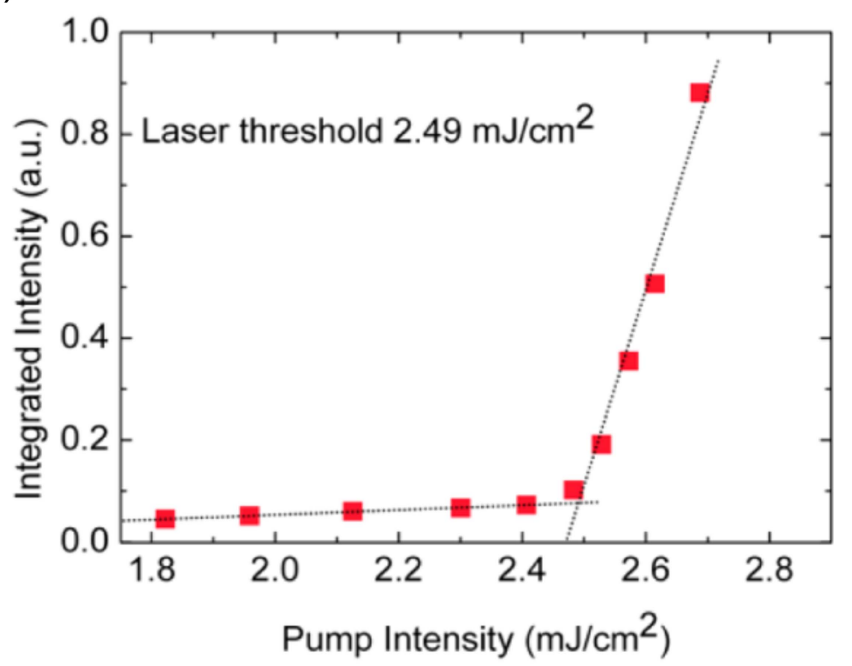

Fig. 8. (a) Schematic of the NPL VCSEL. (b) Emission spectrum of the VCSEL. (c) Integrated emission intensity as a function of pump intensity. The linear lines indicate a lasing threshold of $2.49 \mathrm{~mJ} / \mathrm{cm}^{2}$ under 2-PA pumping. Reprinted with permission from [68], copyright 2014, American Chemical Society.

lasers based on solution-processed materials [105]. The large biexciton binding energy in these NPLs leads to biexciton-assisted ASE with an ultralow ASE threshold of $\sim 6 \mu \mathrm{J} / \mathrm{cm}^{2}$, which is adequate to realize CW-pumped ASE and lasing in microcavities. Furthermore, CW lasing was also demonstrated in a photonic crystal high- $Q$ nanobeam cavity with a record low threshold of $1 \mu \mathrm{W}$ [113]. In this nanolaser, high-efficiency core/shell NPLs were coupled to a high- $Q$ silicon nitride photonic crystal nanobeam cavity, resulting in the record low lasing threshold. The threshold can be further lowered by employing cavities with a higher $Q$ value [114]. These promising results show that NPLs may present a practical alternative to the existing laser gain media.

\section{Solar Energy-Harvesting Applications}

LSCs are light management devices used to concentrate solar flux into Si-based solar cells [123], [124]. The usual LSC design comprises a plastic or glass plate embedded or coated with luminescent emitters. These large-area sunlight collectors absorb incident solar photons through the imbedded luminescent species that generally emit light at higher wavelengths. Furthermore, these LSC slabs guide the emitted photons via total internal reflection to the device edges that are attached to photovoltaic cells. Both direct and diffuse solar radiations can be spatially and energetically concentrated with LSCs, which in turn helps reduce the overall area of solar cells, which is required to meet current energy demands. Among different concentrating devices, LSCs are attracting great attention due to their simple, sustainable, and tunable architecture. LSCs were proposed approximately 40 years ago and have failed to make any industrial impact because of the limited availability of the materials chosen. In the last decade, LSCs have been revitalized with the advancements in the synthesis and improvements in the optical properties of semiconductor inorganic colloidal NCs [125]. Currently, in addition to the solar flux concentration property, LSC 
panels with a semitransparent nature can also be fabricated [125]. For heavily urbanized regions, this semitransparent nature of LSCs in the form of window panels and facades of buildings can offer effective solar energy generation solutions when they are employed as part of the zero-energy building concept [124].

Doping of semiconductor NCs results in an efficient Stokes-shifted emission, which is very much desired for LSC emitter materials. In a recent comparative study, $\mathrm{Cu}$-doped CdSe CQDs were shown to possess high performance among the available luminophores (CdSe/CdS giant QDs, CdSe/CdS dots in rods, and Mn-doped ZnCdS/ZnS CQDs) [89]. Although these doped CQDs possess moderate PL QYs (30\%), the large Stokes shift, NIR emission, and large absorption overlap with the solar spectrum help boost their performance as an LSC material. Cu-doped CdSe NPLs, which were recently introduced into the CQW family, possess extraordinarily high PL QYs (80\%-90\%), dominant and large Stokes-shifted NIR emission, high absorption cross sections, and steplike optical absorption profiles [20]. These exceptional properties enable these materials to be better emitters in LSCs. To harness these extraordinary properties of doped NPLs, we studied in detail the reabsorption properties of these doped NPLs and compared them to the previous best reported doped CQDs. Fig. 9(a) shows the spectrally integrated emission intensities for three different LSC emitters, measured as a function of excitation distance for the concentrated solutions of LSC emitters. Using a fixed power LED, light source excitation was provided at different known lengths, and emission was measured with a fiber-coupled spectrometer at the edge of a quartz pipe. The entire reabsorption measurement setup is shown in Fig. 9(c). A concentrated solution with an optical density of one measured at a $1 \mathrm{~mm}$ thickness was filled into a 1-m-long quartz pipe. Here, Cu-doped CdSe NPLs with vertical thicknesses of 3 and $4 \mathrm{ML}$ are compared with the previous best reported Cu-doped CdSe CQDs [20]. As seen by both theoretical and experimental measurements, 4-ML CdSe NPLs perform better than 3-ML Cu-doped NPLs and CQDs. By following a previously used calculation, the reabsorption probabilities were calculated for all studied emitters, and according to the experimental results, 4-ML Cu-doped NPLs shows a larger value of reduced length [see Fig. 9(b)].

These materials were also tested in a real 2-D plastic LSC matrix, and their performance was also tested with standard Si solar cells [20]. Fig. 9(d) shows the absorption and emission spectra of 3-4 ML Cu-doped NPLs in toluene and embedded in a Poly(lauryl methacrylate) (PLMA) matrix. Moreover, the overlap of NPL absorption with the standard solar simulator AM 1.5G is shown. Fig. 9(e) shows the real images of $10 \times 10 \mathrm{~cm}^{2}$ LSCs made of Cu-doped NPLs under UV and daylight conditions. Finally, the performance of these materials was tested by measuring their optical efficiencies $\left(\eta_{\text {opt }}\right)$ by connecting a Si solar cell on one of the edges of a $10 \times 10 \mathrm{~cm}^{2}$ LSC slab. To reproduce the real situation, no back diffuser or reflector was used in all the measurements. $\eta_{\text {opt }}$ for CQDs, 3-ML LSC slab, and 4ML LSC slab was measured as $1.52 \%, 1.65 \%$, and $1.60 \%$, respectively. Further improvements were envisioned by improving the PL QY of 4-ML-doped NPLs by improving the PLMA matrix and by using advanced heterostructures of doped NPLs, e.g., doped core-crown and core-crownshell.

\section{OUTLOOK AND FUTURE PER S P E C T IV E}

In this review, we discuss the recent developments in the field of colloidal semiconductor NPLs. With the tremendous efforts of the scientific community, the synthesis of CdSe-based NPLs and their heterostructures (e.g., core-shell and core-crown) has matured in recent years. Along with possessing the unique physical properties of 2-D QWs, these solution-processed CdSe-based NPLs have also benefited from the processing advantages of solution-processed materials. The solution processability of NPLs, as discussed earlier, suggests that the nanophotonic devices made from NPLs can be integrated essentially onto any (flexible) substrates that may find application in labon-chip diagnostics systems, chemical sensing, and so on. In the last decade, based on their strict 1-D quantum confinement, which is controllable with atomic precision, these materials were explored for different optoelectronic and nanophotonic applications. With the extensive expertise gained with the exploration of $0-\mathrm{D}$ and 1-D NCs, the colloidal synthesis of these 2-D NCs and their successful utilization in different devices is accelerating. This review has summarized selected examples from the literature, indicating that this new category of optoelectronic materials exhibits several advantages that are promising for establishing high-performance LEDs, lasers, and LSCs. However, several open questions need to be addressed before the widespread utilization of these materials in the nanophotonics industry.

First, for almost all optoelectronic devices, the solutionprocessed NPLs must be successfully transformed into uniform and densely packed solid films. In a solid film form, these anisotropic platelets tend to form long vertical stacks, which drastically limits their performance [74], [126]. Recently, some groups have focused on controlling the self-assembly of 2-D NPLs in the horizontal versus vertical directions [127]-[129]. Thus, for all optoelectronic devices, a solution to this orientation-related problem in NPLs and their successful deposition on any desired substrate with high packing density should be realized while maintaining the high PL QYs in the film form. Second, for LEDs based on NPLs, a very little effort has been made to improve their performance and device stability. To date, no inorganic CTLs have been applied in NPL-based devices. For QD-LEDs, all-inorganic CTLs are shown to possess high performance with improved operational lifetimes. Moreover, with the recent development of near-unity emitting and highly stable core-shell CdSe/ZnS 

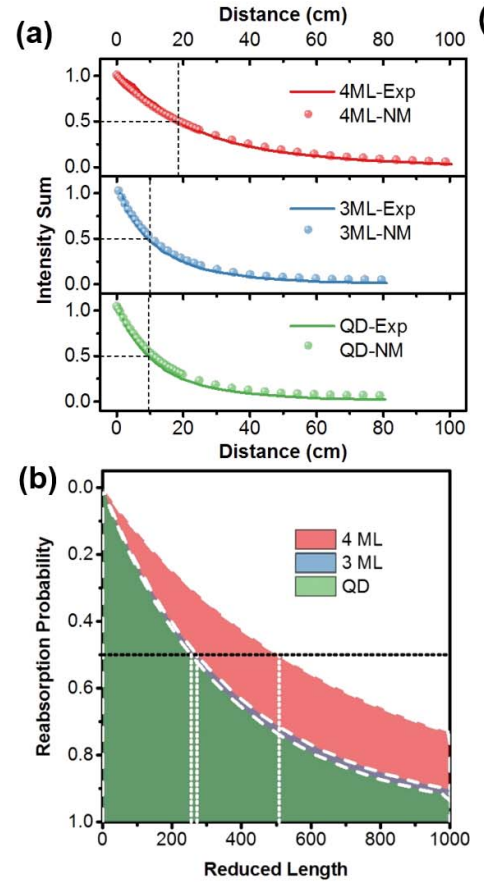

(c)

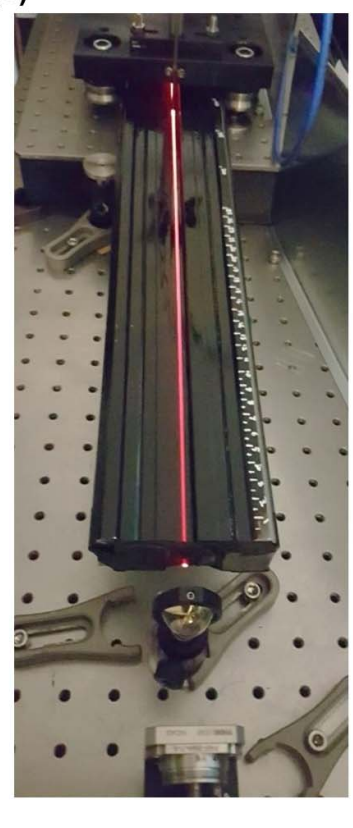

(d)

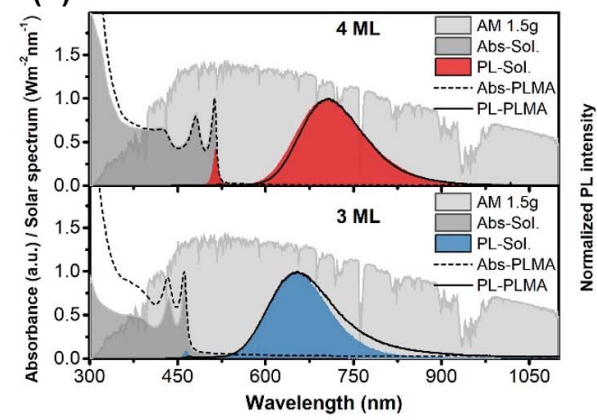

(e)

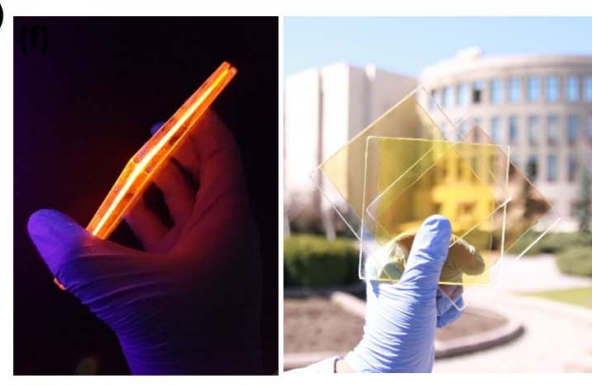

Fig. 9. (a) PL intensities that are spectrally integrated for different luminophores (4- and 3-ML NPLs) as a function of excitation distance from both experiments and numerical modeling. (b) Reabsorption probability versus reduced length. (c) Photograph of the 1-D liquid waveguide setup with a fiber-coupled excitation source and related optics at the collector end. (d) Steady-state absorption and emission spectra of Cu-doped 3-and 4-ML NPLs in the toluene solution and in a PLMA matrix. (e) Real-color photographs of a fabricated $10 \times 10 \mathrm{~cm}^{2}$ LSC with 3-ML NPLs incorporated in a PLMA matrix under UV illumination and similar sized LSCs with Cu-doped 3- and 4-ML NPLs and CQDs in daylight. Reprinted (adapted) with permission from [20] [(a)-(e) copyright 2017, Wiley].

NPLs synthesized by high-temperature shell growth procedures, the performance of NPL-based LEDs is expected to reach close to that of the best reported QD-LEDs, along with high color purities. Reduced Auger recombination in core-shell NPLs can benefit their application in LEDs. Under the high-current operation of these LEDs, the minimization of the Auger rates could improve the overall efficiencies [26].

Third, a very few reports on doping of flat CdSe NPLs have been presented. However, initial reports suggest a great potential for these doped atomically flat NCs. In the case of Cu-doped CdSe NPLs, further shifting of the absorption and emission spectra close to the silicon bandgap will support the successful utilization of these materials in future LSCs. This shift can possibly be achieved by successfully doping core-shell NPLs; however, initial studies suggest that their near-unity PL QYs significantly drop to only $5 \%-10 \%$ after coating with a thin CdS shell. Thus, successful utilization of doped NPLs as potential emitters in LSC applications requires further work aimed at high photostability and sustained PL QYs in core-shell architectures. Very recently, LSCs based on quantum-cutting $\mathrm{Yb}^{3+}: \mathrm{CsPb}\left(\mathrm{Cl}_{1-x} \mathrm{Br}_{x}\right)^{3}$ NCs have been documented with PL QYs as high as 200\% [130], [131]. These materials also possess narrow emission linewidths with a very large Stokes shift that enables spectral matching with Si solar cells. However, to realize a perfect LSC emitter material, the low photostability and low solar absorption remain concerns for the doped perovskite NCs [131], [132]. CdSe-based NPLs and their heterostructures with very high absorption cross sections are the potential candidates for doping with $\mathrm{Yb}^{3+}$, which may lead to similar quantumcutting-enabled high PL QYs along with tunable and higher solar absorption.

The well-investigated field of application for flat NPLs in recent years is the high-performance ASE and lasing. In the last five years, NPLs have been shown to be very good gain materials with low-gain thresholds and high-gain coefficients. CW lasing at room temperature was successfully shown by incorporating core-shell NPLs into photonic crystal cavities with small mode volumes. These initial results showed the best performance among all the reported studies on room-temperature lasing. More importantly, these lasing thresholds are almost always limited by the quality factor of the cavity. Therefore, further improvements by improving the quality factor of photonic crystals are projected. Thus, the extreme color purity and reduced Auger rates for core-shell NPLs with excellent lasing properties make these materials potential candidates for future lasing applications. The broad emission tunability of CdSe NPLs would enable lasers with multiple colors, which are difficult to realize using semiconductor and solid-state lasers. The scientific community envisions that future work in this field will involve improving the stability of NPL materials 
via suitable surface treatments and better encapsulation of the devices and achieving lasing through electrical injection rather than optical pumping.

In conclusion, future studies in the field of semiconductor NPLs should continue to build a library of atomically flat NCs that include improved CdSe-based NPLs and their advanced heterostructures as well as cadmium-free 2-D NPLs (e.g., ZnSe, ZnS, CuInSe, and their heterostructures) to completely understand their interesting and useful photophysical properties and to successfully utilize these materials in different optoelectronic applications. However, in order to become a practically viable option, the other forms of 2-D NPLs should possess good enough optoelectronic performance and preferably surpass or at least match that of current CdSe-based NPLs and their heterostructures. Current and envisioned advances in the nanophotonics field would enable colloidal semiconductor CdSe-based NPLs to be a new paradigm for solid-state material discovery and analysis.

\section{Acknowledgment}

H. V. Demir would like to thank the Turkish National Academy of Sciences (TUBA). The authors declare no competing financial interest.

\section{REFERENCES}

[1] A. I. Ekimov and A. A. Onushchenko, "Quantum size effect in three-dimensional microscopic semiconductor crystals," JETP Lett., vol. 34, no. 6, pp. 345-349, 1981.

[2] L. E. Brus, "A simple model for the ionization potential, electron affinity, and aqueous redox potentials of small semiconductor crystallites," J. Chem. Phys., vol. 79, no. 11, pp. 5566-5571, 1983.

[3] C. B. Murray, D. J. Norris, and M. G. Bawendi, "Synthesis and characterization of nearly monodisperse CdE (E = sulfur, selenium, tellurium) semiconductor nanocrystallites," J. Amer. Chem. Soc., vol. 115, no. 19, pp. 8706-8715, 1993.

[4] J. F. Suyver, T. van der Beek, S. F. Wuister J. J. Kelly, and A. Meijerink, "Luminescence of nanocrystalline $\mathrm{ZnSe}$ :Cu," Appl. Phys. Lett., vol. 79, no. 25, pp. 4222-4224, 2001.

[5] X. Peng et al., "Shape control of CdSe nanocrystals," Nature, vol. 404, no. 6773 , pp. 59-61, 2000.

[6] M. C. Schlamp, X. Peng, and A. P. Alivisatos, "Improved efficiencies in light emitting diodes made with CdSe(CdS) core/shell type nanocrystals and a semiconducting polymer," J. Appl. Phys., vol. 82, no. 11, pp. 5837-5842, 1997.

[7] M. Lomascolo, A. Cretí, G. Leo, L. Vasanelli, and L. Manna, "Exciton relaxation processes in colloidal core/shell ZnSe/ZnS nanocrystals," Appl. Phys. Lett., vol. 82, no. 3, pp. 418-420, 2003.

[8] S.-Y. Lu, M.-L. Wu, and H.-L. Chen, "Polymer nanocomposite containing CdS-ZnS core-shell particles: Optical properties and morphology," J. Appl. Phys., vol. 93, no. 9, pp. 5789-5793, 2003.

[9] K. Boldt, K. N. Schwarz, N. Kirkwood, T. A. Smith, and P. Mulvaney, "Electronic structure engineering in ZnSe/CdS type-II nanoparticles by interface alloying," J. Phys. Chem. C, vol. 118, no. 24, pp. 13276-13284, 2014.

[10] D. V. Talapin, J. H. Nelson, E. V. Shevchenko, S. Aloni, B. Sadtler, and A. P. Alivisatos, "Seeded growth of highly luminescent CdSe/CdS nanoheterostructures with rod and tetrapod morphologies," Nano Lett., vol. 7, no. 10, pp. 2951-2959, 2007.

[11] J. Joo, J. S. Son, S. G. Kwon, J. H. Yu, and T. Hyeon, "Low-temperature solution-phase synthesis of quantum well structured CdSe nanoribbons," J. Amer. Chem. Soc., vol. 128 no. 17 , pp. $5632-5633,2006$

[12] S. Ithurria and B. Dubertret, "Quasi 2D colloidal CdSe platelets with thicknesses controlled at the atomic level," J. Amer. Chem. Soc., vol. 130 , no. 49, pp. 16504-16505, 2008.

[13] S. Ithurria, M. D. Tessier, B. Mahler, R. P. S. M. Lobo, B. Dubertret, and A. L. Efros, "Colloidal nanoplatelets with two-dimensional electronic structure," Nature Mater., vol. 10, no. 12, pp. 936-941, Dec. 2011

[14] C. Bouet, M. D. Tessier, S. Ithurria, B. Mahler,
B. Nadal, and B. Dubertret, "Flat colloidal semiconductor nanoplatelets," Chem. Mater. vol. 25, no. 8, pp. 1262-1271, 2013.

[15] S. Pedetti, S. Ithurria, H. Heuclin, G. Patriarche, and B. Dubertret, "Type-II CdSe/CdTe core/crown semiconductor nanoplatelets," J. Amer. Chem. Soc., vol. 136, no. 46, pp. 16430-16438, 2014.

[16] Y. Kelestemur, B. Guzelturk, O. Erdem, M. Olutas, K. Gungor, and

H. V. Demir, "Platelet-in-box colloidal quantum wells: CdSe/CdS CdS core/crown shell heteronanoplatelets," Adv. Funct. Mater., vol. 26, no. 21, pp. 3570-3579, 2016.

[17] Y. Kelestemur, M. Olutas, S. Delikanli, B. Guzelturk, M. Z. Akgul, and H. V. Demir "Type-II colloidal quantum wells: CdSe/CdT core/crown heteronanoplatelets," J. Phys. Chem. C, vol. 119, pp. 2177-2185, Jan. 2015.

[18] Y. Gao et al., "Low-threshold lasing from colloidal $\mathrm{CdSe} / \mathrm{CdSeTe}$ core/alloyed-crown type-II heteronanoplatelets," Nanoscale, vol. 10, no. 20, pp. 9466-9475, 2018.

[19] L. Biadala, F. Liu, M. D. Tessier, D. R. Yakovlev, B. Dubertret, and M. Bayer, "Recombination dynamics of band edge excitons in quasi-two-dimensional CdSe nanoplatelets," Nano Lett., vol. 14, no. 3, pp. 1134-1139, 2014.

[20] M. Sharma et al., "Near-unity emitting copper-doped colloidal semiconductor quantum wells for luminescent solar concentrators," Adv Mater., vol. 29, no. 30, Aug. 2017, Art. no. 1700821.

[21] M. Sharma et al., "Understanding the journey of dopant copper ions in atomically flat colloidal nanocrystals of CdSe nanoplatelets using partial cation exchange reactions," Chem. Mater., vol. 30 no. 10 , pp. 3265-3275, 2018.

[22] S. Delikanli et al., "Mn2 ${ }^{+}$-doped CdSe/CdS core/multishell colloidal quantum wells enabling tunable carrier-dopant exchange interactions," ACS Nano, vol. 9, no. 12, pp. 12473-12479, 2015.

[23] A. H. Khan et al., "Tunable and efficient red to near-infrared photoluminescence by synergistic exploitation of core and surface silver doping of CdSe nanoplatelets," Chem. Mater., vol. 31, no. 4 pp. 1450-1459, 2019.

[24] B. Martinez et al., "Doping as a strategy to tune color of 2D colloidal nanoplatelets," ACS Appl. Mater. Interfaces, vol. 11, no. 10 pp. 10128-10134, 2019.

[25] T. Galle et al., "Colloidal mercury-doped CdSe nanoplatelets with dual fluorescence," Chem. Mater, vol. 31, no. 14, pp. 5065-5074, 2019.

[26] M. Pelton, "Carrier dynamics, optical gain, and lasing with colloidal quantum wells," $J$. Phys. Chem. C, vol. 122, no. 20, pp. 10659-10674, 2018.

[27] Y. Wang et al., "Stimulated emission and lasing from CdSe/CdS/ZnS core-multi-shell quantum dots by simultaneous three-photon absorption," Adv. Mater, vol. 26, no. 18, pp. 2954-2961, May 2014.
[28] Z. Chen, B. Nadal, B. Mahler, H. Aubin, and B. Dubertret, "Quasi-2D colloidal semiconductor nanoplatelets for narrow electroluminescence," Adv. Funct. Mater., vol. 24, no. 3, pp. 295-302, Jan. 2014.

[29] E. Lhuillier, S. Pedetti, S. Ithurria, B. Nadal, H. Heuclin, and B. Dubertret, "Two-dimensiona colloidal metal chalcogenides semiconductors: Synthesis, spectroscopy, and applications," Accounts Chem. Res., vol. 48, no. 1, pp. 22-30, 2015

[30] J. S. Son et al., "Large-scale soft colloidal template synthesis of $1.4 \mathrm{~nm}$ thick CdSe nanosheets," Angew. Chem. Int. Ed., vol. 48, no. 37, pp. 6993-6996, Sep. 2009.

[31] J. S. Son et al., "Dimension-controlled synthesis of CdS nanocrystals: From OD quantum dots to 2D nanoplates," Small, vol. 8, no. 15, pp. 2394-2402, 2012

[32] S. Ithurria, G. Bousquet, and B. Dubertret, "Continuous transition from 3D to 1D confinement observed during the formation of CdSe nanoplatelets," J. Amer. Chem. Soc., vol. 133, no. 9, pp. 3070-3077, 2011

[33] A. Riedinger et al., "An intrinsic growth instability in isotropic materials leads to quasi-two-dimensional nanoplatelets," Nature Mater., vol. 16, pp. 743-749, Jul. 2017.

[34] M. D. Tessier, C. Javaux, I. Maksimovic, V. Loriette, and B. Dubertret, "Spectroscopy of single CdSe nanoplatelets," ACS Nano, vol. 6, no. 8, pp. 6751-6758, 2012

[35] S. Christodoulou et al., "Chloride-induced thickness control in CdSe nanoplatelets," Nano Lett., vol. 18, no. 10, pp. 6248-6254, 2018

[36] A. Chu, C. Livache, S. Ithurria, and E. Lhuillier, "Electronic structure robustness and design rules for 2D colloidal heterostructures," J. Appl. Phys., vol. 123 , no. 3, 2018, Art. no. 035701

[37] S. Ithurria and D. V. Talapin, "Colloidal atomic layer deposition (c-ALD) using self-limiting reactions at nanocrystal surface coupled to phase transfer between polar and nonpolar media," J. Amer. Chem. Soc., vol. 134, no. 45, pp. 18585-18590, 2012

[38] C. She et al., "Red, yellow, green, and blue amplified spontaneous emission and lasing using colloidal CdSe nanoplatelets," ACS Nano, vol. 9, no. 10, pp. 9475-9485, 2015.

[39] A. Yeltik, S. Delikanli, M. Olutas, Y. Kelestemur B. Guzelturk, and H. V. Demir, "Experimental determination of the absorption cross-section and molar extinction coefficient of colloidal CdSe nanoplatelets," J. Phys. Chem. C, vol. 119, no. 47, pp. 26768-26775, 2015

[40] A. W. Achtstein, A. Antanovich, A. Prudnikau, U. Woggon, and M. Artemyev, "Linear absorption in CdSe nanoplates: Thickness and lateral size dependency of the intrinsic absorption," J. Phys. Chem. C, vol. 119, pp. 20156-20161, Aug. 2015.

[41] C. She et al., "Low-threshold stimulated emission using colloidal quantum wells," Nano Lett., vol. 14, no. 5, pp. 2772-2777, 2014. 
[42] S. Delikanli, G. Yu, A. Yeltik, S. Bose, T. Erdem, and J. Yu, "Ultrathin highly luminescent two-monolayer colloidal CdSe nanoplatelets," Adv. Funct. Mater., vol. 29, Aug. 2019, Art. no. 1901028.

[43] M. A. Hines and P. Guyot-Sionnest, "Synthesis and characterization of strongly luminescing ZnS-capped CdSe nanocrystals," J. Phys. Chem., vol. 100, no. 2, pp. 468-471, 1996.

[44] B. Mahler, P. Spinicelli, S. Buil, X. Quelin, J.-P. Hermier, and B. Dubertret, "Towards non-blinking colloidal quantum dots," Nature Mater., vol. 7, no. 8, pp. 659-664, 2008.

[45] J. L. Casson et al., "'Giant' multishell CdSe nanocrystal quantum dots with suppressed blinking," J. Amer. Chem. Soc., vol. 130, no. 15, pp. 5026-5027, 2008.

[46] O. Chen et al., "Compact high-quality CdSe-CdS core-shell nanocrystals with narrow emission linewidths and suppressed blinking," Nature Mater., vol. 12, no. 5, pp. 445-451, 2013.

[47] Y. He et al., "Microwave synthesis of water-dispersed CdTe/CdS/ZnS core-shell-shell quantum dots with excellent photostability and biocompatibility," Adv. Mater., vol. 20, no. 18, pp. 3416-3421, 2008.

[48] X. Dai et al., "Solution-processed, high-performance light-emitting diodes based on quantum dots," Nature, vol. 515, no. 6, pp. 96-99, Nov. 2014.

[49] C. Dang, J. Lee, C. Breen, J. S. Steckel, S. Coe-Sullivan, and A. Nurmikko, "Red, green and blue lasing enabled by single-exciton gain in colloidal quantum dot films," Nature Nanotechnol., vol. 7, no. 5, pp. 335-339, 2012.

[50] X. Michalet et al., "Quantum dots for live cells, in vivo imaging, and diagnostics," Science, vol. 307, pp. 538-545, Jan. 2005.

[51] B. Mahler, B. Nadal, C. Bouet, G. Patriarche, and B. Dubertret, "Core/shell colloidal semiconductor nanoplatelets," J. Amer. Chem. Soc., vol. 134, no. 45, pp. 18591-18598, 2012.

[52] S. Shendre et al., "Ultrahigh-efficiency aqueous flat nanocrystals of CdSe/CdS@Cd $\mathrm{Cd}_{1-x} \mathrm{Zn}_{x} \mathrm{~S}$ colloidal core/crown@alloyed-shell quantum wells," Nanoscale, vol. 11, no. 1, pp. 301-310, 2019.

[53] F. Muckel et al., "Sp-d exchange interactions in wave function engineered colloidal CdSe/Mn:CdS hetero-nanoplatelets," Nano Lett., vol. 18, no. 3, pp. 2047-2053, 2018.

[54] J. R. Murphy et al., "Time-resolved photoluminescence study of CdSe/CdMnS/CdS core/multi-shell nanoplatelets," Appl. Phys. Lett., vol. 108, no. 24, 2016, Art. no. 242406.

[55] M. D. Tessier, B. Mahler, B. Nadal, H. Heuclin, S. Pedetti, and B. Dubertret, "Spectroscopy of colloidal semiconductor core/shell nanoplatelets with high quantum yield," Nano Lett., vol. 13 no. 7, pp. 3321-3328, 2013.

[56] A. Polovitsyn et al., "Synthesis of air-stable CdSe/ZnS core-shell nanoplatelets with tunable emission wavelength," Chem. Mater., vol. 29, no. 13, pp. 5671-5680, 2017.

[57] A. A. Rossinelli, A. Riedinger, P. Marqués-Gallego, P. N. Knüsel, F. V. Antolinez, and D. J. Norris, "High-temperature growth of thick-shell CdSe/CdS core/shell nanoplatelets," Chem. Commun., vol. 53, no. 71, pp. 9938-9941, 2017.

[58] Y. Altintas et al., "Highly stable, near-unity efficiency atomically flat semiconductor nanocrystals of CdSe/ZnS hetero-nanoplatelets enabled by ZnS-shell hot-injection growth," Small, vol. 15 , no. 8, 2019, Art. no. 1804854

[59] F. Feng et al., "Probing the fluorescence dipoles of single cubic CdSe/CdS nanoplatelets with vertical or horizontal orientations," ACS Photon., vol. 5, no. 5, pp. 1994-1999, 2018.

[60] K. Boldt, N. Kirkwood, G. A. Beane, and P. Mulvaney, "Synthesis of highly luminescent and photo-stable, graded shell CdSe/Cd $\mathrm{Zn}_{1-x} \mathrm{~S}$ nanoparticles by in situ alloying," Chem. Mater., vol. 25, no. 23, pp. 4731-4738, 2013.
[61] G. Sirigu et al., "Dual emission and optical gain in $\mathrm{PbS} / \mathrm{CdS}$ nanocrystals: Role of shell volume and of core/shell interface," Phys. Rev. B, Condens. Matter, vol. 96, no. 15, 2017, Art. no. 155303.

[62] V. I. Klimov et al., "Single-exciton optical gain in semiconductor nanocrystals," Nature, vol. 447. no. 7143, pp. 441-446, May 2007.

[63] J. M. Pietryga et al., "Spectroscopic and device aspects of nanocrystal quantum dots," Chem. Rev., vol. 116, no. 18, pp. 10513-10622, 2016.

[64] S. Bisschop, P. Geiregat, T. Aubert, and Z. Hens, "The impact of core/shell sizes on the optical gain characteristics of CdSe/CdS quantum dots," ACS Nano, vol. 12, no. 9, pp. 9011-9021, 2018.

[65] M. D. Tessier, P. Spinicelli, D. Dupont, G. Patriarche, S. Ithurria, and B. Dubertret, "Efficient exciton concentrators built from colloidal core/crown CdSe/CdS semiconductor nanoplatelets," Nano Lett., vol. 14, no. 1, pp. 207-213, 2014.

[66] A. Prudnikau, A. Chuvilin, and M. Artemyev, "CdSe-CdS nanoheteroplatelets with efficient photoexcitation of central CdSe region through epitaxially grown CdS wings," J. Amer. Chem. Soc., vol. 135, no. 39, pp. 14476-14479, 2013.

[67] F. Zhang et al., "Super color purity green quantum dot light-emitting diodes fabricated by using CdSe/CdS nanoplatelets," Nanoscale, vol. 8 , no. 24, pp. 12182-12188, 2016.

[68] B. Guzelturk, Y. Kelestemur, M. Olutas, S. Delikanli, and H. V. Demir, "Amplified spontaneous emission and lasing in colloidal nanoplatelets," ACS Nano, vol. 8, no. 7, pp. 6599-6605, 2014.

[69] S. Delikanli et al., "Continuously tunable emission in inverted type-I CdS/CdSe core/crown semiconductor nanoplatelets," Adv. Funct. Mater. vol. 25, no. 27, pp. 4282-4289, 2015

[70] Y. Kelestemur et al., "CdSe/CdSe ${ }_{1-x} \mathrm{Te}_{x}$ core/crown heteronanoplatelets: Tuning the excitonic properties without changing the thickness," J. Phys. Chem. C, vol. 121, no. 8 , pp. 4650-4658, 2017.

[71] B. Liu, S. Delikanli, Y. Gao, D. Dede, K. Gungor, and H. V. Demir, "Nanocrystal light-emitting diodes based on type II nanoplatelets," Nano Energy, vol. 47, pp. 115-122, Mar. 2018.

[72] B. Guzelturk, Y. Kelestemur, M. Olutas, Q. Li, T. Lian, and H. V. Demir, "High-efficiency optical gain in type-II semiconductor nanocrystals of alloyed colloidal quantum wells," J. Phys. Chem. Lett., vol. 8, no. 21, pp. 5317-5324, 2017.

[73] T. Kodanek, H. M. Banbela, S. Naskar, P. Adel, N. C. Bigall, and D. Dorfs, "Phase transfer of 1- and 2-dimensional Cd-based nanocrystals," Nanoscale, vol. 7, no. 45, pp. 19300-19309, 2015.

[74] W. D. Kim et al., "Pushing the efficiency envelope for semiconductor nanocrystal-based electroluminescence devices using anisotropic nanocrystals," Chem. Mater., vol. 31, no. 9, pp. 3066-3082, 2019.

[75] D. Dede et al., "Highly stable multicrown heterostructures of type-II nanoplatelets for ultralow threshold optical gain," Chem. Mater. vol. 31, no. 5, pp. 1818-1826, 2019.

[76] A. M. Schimpf, K. E. Knowles, G. M. Carroll, and D. R. Gamelin, "Electronic doping and redox-potential tuning in colloidal semiconductor nanocrystals," Accounts Chem. Res., vol. 48, no. 7 pp. 1929-1937, 2015.

[77] A. Hazarika et al., "Ultranarrow and widely tunable $\mathrm{Mn}^{2+}$-induced photoluminescence from single Mn-doped nanocrystals of ZnS-CdS alloys,' Phys. Rev. Lett., vol. 110, no. 26, 2013, Art. no. 267401

[78] A. Hazarika, A. Pandey, and D. D. Sarma, "Rainbow emission from an atomic transition in doped quantum dots," J. Phys. Chem. Lett., vol. 5 , no. 13, pp. 2208-2213, 2014.

[79] G. K. Grandhi, R. Tomar, and R. Viswanatha, "Study of surface and bulk electronic structure of II-VI semiconductor nanocrystals using $\mathrm{Cu}$ as a nanosensor," ACS Nano, vol. 6 , no. 11 , pp. 9751-9763, 2012

[80] B. B. Srivastava, S. Jana, and N. Pradhan, "Dopin $\mathrm{Cu}$ in semiconductor nanocrystals: Some old and some new physical insights," J. Amer. Chem. Soc., vol. 133, no. 4, pp. 1007-1015, 2011.

[81] M. Sharma, S. Singh, and O. P. Pandey, "Excitation induced tunable emission in biocompatible chitosan capped ZnS nanophosphors," J. Appl. Phys., vol. 107, no. 10, 2010, Art. no. 104319.

[82] A. Singh, R. Kaur, O. P. Pandey, X. Wei, and M. Sharma, "Synthesis of fluorescent core-shell nanomaterials and strategies to generate white light," J. Appl. Phys., vol. 118, no. 4, 2015, Art. no. 044305.

[83] M. Mittal, M. Sharma, and O. P. Pandey, "Fast and quick degradation properties of doped and capped ZnO nanoparticles under UV-visible light radiations," Sol. Energy, vol. 125, pp. 51-64, Feb. 2016.

[84] M. Kaur et al., "Cd-free Cu-doped ZnInS/ZnS core/shell nanocrystals: Controlled synthesis and photophysical properties," Nanosc. Res. Lett., vol. 13, no. 1, p. 182, 2018

[85] S. Jana, B. B. Srivastava, and N. Pradhan, "Correlation of dopant states and host bandgap in dual-doped semiconductor nanocrystals," J. Phys. Chem. Lett., vol. 2, no. 14, pp. 1747-1752, 2011.

[86] S. Jana, G. Manna, B. B. Srivastava, and N. Pradhan, "Tuning the emission colors of semiconductor nanocrystals beyond their bandgap tunability: All in the dope," Small, vol. 9, no. 22, pp. 3753-3758, Nov. 2013.

[87] J. T. Wright and R. W. Meulenberg, "Effects of dopants on the band structure of quantum dots: A theoretical and experimental study," Phys. Rev. B, Condens. Matter, vol. 88, no. 4, 2013, Art. no. 045432

[88] L. Yang, K. E. Knowles, A. Gopalan, K. E. Hughes, M. C. James, and D. R. Gamelin, "One-pot synthesis of monodisperse colloidal copper-doped CdSe nanocrystals mediated by ligand-copper interactions," Chem. Mater., vol. 28, no. 20, pp. 7375-7384, 2016

[89] L. R. Bradshaw, K. E. Knowles, S. McDowall, and D. R. Gamelin, "Nanocrystals for luminescent solar concentrators," Nano Lett., vol. 15, no. 2, pp. 1315-1323, Feb. 2015

[90] G. K. Grandhi and R. Viswanatha, "Tunable infrared phosphors using $\mathrm{Cu}$ doping in semiconductor nanocrystals: Surface electronic structure evaluation," J. Phys. Chem. Lett., vol. 4, no. 3, pp. 409-415, 2013

[91] J. T. Wright, K. Forsythe, J. Hutchins, and R. W. Meulenberg, "Implications of orbital hybridization on the electronic properties of doped quantum dots: The case of Cu:CdSe," Nanoscale, vol. 8, no. 17, pp. 9417-9424, 2016.

[92] R. Tenne et al., "From dilute isovalent substitution to alloying in CdSeTe nanoplatelets," Phys. Chem. Chem. Phys., vol. 18, no. 22, pp. 15295-15303, 2016.

[93] V. Wood and V. Bulović, "Colloidal quantum dot light-emitting devices.," Nano Rev., vol. 1, pp. 1-7, Jan. 2010.

[94] Y. Shirasaki, G. J. Supran, M. G. Bawendi, and V. Bulovic, "Emergence of colloidal quantum-dot light-emitting technologies," Nature Photon. vol. 7, pp. 13-23, Dec. 2013.

[95] M. K. Choi, J. Yang, T. Hyeon, and D.-H. Kim, "Flexible quantum dot light-emitting diodes for next-generation displays," NPJ Flexible Electron. vol. 2, no. 1, p. 10, 2018.

[96] J. Yang, M. K. Choi, D.-H. Kim, and T. Hyeon, "Designed assembly and integration of colloidal nanocrystals for device applications," Adv. Mater. vol. 28, pp. 1176-1207, Feb. 2016.

[97] V. L. Colvin, M. C. Schlamp, and A. P. Allvlsatos, "Light-emitting diodes made from cadmium selenide nanocrystals and a semiconducting polymer," Nature, vol. 370, pp. 354-357, Aug. 1994.

[98] U. Giovanella et al., "Efficient solution-processed 
nanoplatelet-based light-emitting diodes with high operational stability in air," Nano Lett. vol. 18, no. 6, pp. 3441-3448, 2018.

[99] K. Saxena, V. K. Jain, and D. S. Mehta, "A review on the light extraction techniques in organic electroluminescent devices," Opt. Mater., vol. 32, no. 1, pp. 221-233, 2009.

[100] M.-H. Park et al., "Flexible organic light-emitting diodes for solid-state lighting," J. Photon. Energy, vol. 5, no. 1, 2015, Art. no. 053599.

[101] P. Xiao et al., "Emergence of nanoplatelet light-emitting diodes," Materials, vol. 11, no. 8, p. 1376, 2018.

[102] F. Fan et al., "Colloidal CdSe ${ }_{1-x} \mathrm{~S}_{x}$ nanoplatelets with narrow and continuously-tunable electroluminescence," Nano Lett., vol. 15, no. 7, pp. 4611-4615, 2015.

[103] A. G. Vitukhnovsky, V. S. Lebedev, A. S. Selyukov, A. A. Vashchenko, R. B. Vasiliev, and M. S. Sokolikova, "Electroluminescence from colloidal semiconductor CdSe nanoplatelets in hybrid organic-inorganic light emitting diode," Chem. Phys. Lett., vol. 619, pp. 185-188, Jan. 2015.

[104] P. O. Anikeeva, J. E. Halpert, M. G. Bawendi, and V. Bulovic, "Quantum dot light-emitting devices with electroluminescence tunable over the entire visible spectrum," Nano Lett., vol. 9, no. 7, pp. 2532-2536, Jun. 2009.

[105] J. Q. Grim et al., "Continuous-wave biexciton lasing at room temperature using solution-processed quantum wells," Nature Nanotechnol., vol. 9, no. 11, pp. 891-895, 2014.

[106] M. Pelton, S. Ithurria, R. D. Schaller D. S. Dolzhnikov, and D. V. Talapin, "Carrier cooling in colloidal quantum wells," Nano Lett., vol. 12, no. 12, pp. 6158-6163, 2012.

[107] Y. Kelestemur, D. Dede, K. Gungor, C. F. Usanmaz, O. Erdem, and H. V. Demir, "Alloyed heterostructures of $\mathrm{CdSe}_{x} \mathrm{~S}_{1-x}$ nanoplatelets with highly tunable optical gain performance, Chem. Mater., vol. 29, no. 11 , pp. $4857-4865$, 2017.

[108] B. Guzelturk, Y. Kelestemur, M. Z. Akgul, V. K. Sharma, and H. V. Demir, "Ultralow threshold one-photon- and two-photon-pumped optical gain media of blue-emitting colloidal quantum dot films," J. Phys. Chem. Lett., vol. 5, no. 13, pp. 2214-2218, 2014.

[109] B. T. Diroll, D. V. Talapin, and R. D. Schaller, "Violet-to-blue gain and lasing from colloidal CdS nanoplatelets: Low-threshold stimulated emission despite low photoluminescence quantum yield," ACS Photon., vol. 4, no. 3, pp. 576-583, 2017.

[110] M. Pelton, J. J. Andrews, I. Fedin, D. V. Talapin, H. Leng, and S. K. O'Leary, "Nonmonotonic dependence of Auger recombination rate on shell thickness for CdSe/CdS core/shell nanoplatelets, Nano Lett., vol. 17, no. 11, pp. 6900-6906, 2017.

[111] B. Guzelturk, M. Pelton, M. Olutas, and H. V. Demir, "Giant modal gain coefficients in colloidal II-VI nanoplatelets," Nano Lett., vol. 19 , pp. 277-282, Dec. 2018.

[112] M. Li et al., "Ultralow-threshold multiphoton-pumped lasing from colloidal nanoplatelets in solution," Nature Commun. vol. 6, Sep. 2015, Art. no. 8513.

[113] Z. Yang, M. Pelton, I. Fedin, D. V. Talapin, and E. Waks, "A room temperature continuous-wave nanolaser using colloidal quantum wells," Nature Commun., vol. 8, no. 1, 2017, Art. no. 143.

[114] Q. Quan, P. B. Deotare, and M. Loncar, "Photonic crystal nanobeam cavity strongly coupled to the feeding waveguide," Appl. Phys. Lett., vol. 96, no. 20, 2010, Art. no. 203102

[115] W. W. Chow, A. F. Wright, A. Girndt, F. Jahnke, and S. W. Koch, "Microscopic theory of gain for an InGaN/AlGaN quantum well laser," Appl. Phys. Lett., vol. 71, no. 18, pp. 2608-2610, 1997.

[116] K. Bohnert, G. Schmieder, and C. Klingshirn, "Gain and reflection spectroscopy and the present understanding of the electron-hole plasma in II-VI compounds," Phys. Status Solidi B, vol. 98 , no. 1, pp. 175-188, 1980

[117] M. Kazes, D. Y. Lewis, Y. Ebenstein, T. Mokari, and $\mathrm{U}$. Banin, "Lasing from $\mathrm{CdSe} / \mathrm{ZnS}$ quantum rods in a cylindrical microcavity," Adv. Mater., vol. 14, no. 4, pp. 317-321, 2002

[118] A. V. Malko et al., "From amplified spontaneous emission to microring lasing using nanocrystal quantum dot solids," Appl. Phys. Lett., vol. 81, no. 7, pp. 1303-1305, 2002

[119] D. Geskus, S. Aravazhi, S. M. García-Blanco, and M. Pollnau, "Giant optical gain in a rare-earth-ion-doped microstructure," Adv. Mater. vol. 24 , no. 10 , pp. $22-25,2012$

[120] B. R. Sutherland et al., "Perovskite thin films via atomic layer deposition," Adv. Mater., vol. 27 no. 1, pp. 53-58, 2015

[121] R. Dingle, K. L. Shaklee, R. F. Leheny, and R. B. Zetterstrom, "Stimulated emission and laser action in gallium nitride," Appl. Phys. Lett., vol. 19 , no. 1, pp. 5-7, 1971.
[122] Y.-S. Park, W. K. Bae, T. Baker, J. Lim, and V. I. Klimov, "Effect of Auger recombination on lasing in heterostructured quantum dots with engineered core/shell interfaces," Nano Lett. vol. 15, no. 11, pp. 7319-7328, 2015.

[123] M. G. Debije and P. P. C. Verbunt, "Thirty years of luminescent solar concentrator research: Solar energy for the built environment," Adv. Energy Mater, vol. 2, no. 1, pp. 12-35, 2012.

[124] F. Meinardi, F. Bruni, and S. Brovelli, "Luminescent solar concentrators for building-integrated photovoltaics," Nature Rev. Mater., vol. 2, Dec. 2017, Art. no. 17072.

[125] R. Mazzaro and A. Vomiero, "The renaissance of luminescent solar concentrators: The role of inorganic nanomaterials," Adv. Energy Mater. vol. 8, Nov. 2018, Art. no. 1801903

[126] E. Lhuillier et al., "Electrolyte-gated field effect transistor to probe the surface defects and morphology in films of thick CdSe colloida nanoplatelets," ACS Nano, vol. 8, no. 4 , pp. 3813-3820, 2014.

[127] B. Guzelturk, O. Erdem, M. Olutas, Y. Kelestemur, and H. V. Demir, "Stacking in colloidal nanoplatelets: Tuning excitonic properties," ACS Nano, vol. 8, no. 12, pp. 12524-12533, 2014.

[128] B. Abécassis, M. D. Tessier, P. Davidson, and B. Dubertret, "Self-assembly of CdSe nanoplatelet into giant micrometer-scale needles emitting polarized light," Nano Lett., vol. 14, no. 2, pp. 710-715, 2014

[129] O. Erdem et al., "Orientation-controlled nonradiative energy transfer to colloidal nanoplatelets: Engineering dipole orientation factor," Nano Lett., vol. 19, no. 7, pp. 4297-4305, 2019.

[130] T. J. Milstein, D. M. Kroupa, and D. R. Gamelin, "Picosecond quantum cutting generates photoluminescence quantum yields over $100 \%$ in ytterbium-doped $\mathrm{CsPCl}_{3}$ nanocrystals," Nano Lett., vol. 18, no. 6, pp. 3792-3799, 2018.

[131] T. A. Cohen, T. J. Milstein, D. M. Kroupa J. D. MacKenzie, C. K. Luscombe, and D. R. Gamelin, "Quantum-cutting $\mathrm{Yb}^{3+}$-doped perovskite nanocrystals for monolithic bilayer luminescent solar concentrators," J. Mater. Chem. A, vol. 7, no. 15, pp. 9279-9288, 2019.

[132] R. An et al., "Photostability and photodegradation processes in colloidal $\mathrm{CsPb}_{3}$ perovskite quantum dots," ACS Appl. Mater. Interfaces, vol. 10, no. 45, pp. 39222-39227, 2018

\section{ABOUT THE AUTHORS}

Manoj Sharma received the B.Sc. degree from Punjabi University, Patiala, India, in 2003, the M.Sc. degree from Guru Nanak Dev University, Amritsar, India, in 2005, and the Ph.D. degree from Thapar University, Patiala, in 2011

He was an Assistant Professor with Sri Guru Granth Sahib World University Fatehgarh Sahib, India, from 2011 to 2015 Thereafter, he moved to the National Nanotechnology Research Centre (UNAM), Bilkent University, Ankara, Turkey, as a Visiting Scientist funded by TUBITAK-BIDEB. Since 2017, he has been a Research Fellow with the LUMINOUS! Center of Excellence for Semiconductor Lighting and Displays, School of Electrical and Electronic Engineering, Nanyang Technological University, Singapore. His current research interests include the understanding of light-matter interactions for semiconductor nanocrystals, the synthesis and photophysical studies of colloidal quantum dots, and nanoplatelets for their application in different optoelectronic devices. He has published over $30 \mathrm{SCl}$ journal articles. He presented his work at several international conferences as contributed/invited talks.

Dr. Sharma is a Lifetime Member of the Materials Research Society of India (MRSI) and the Luminescence Society of India (LSI).
Savas Delikanli received the Ph.D. degree in physics from The State University of New York at Buffalo Buffalo, NY, USA, in 2011.

He continued his research as a Postdoctoral Researcher at SUNY at Buffalo until 2013. Then, he was a Scientist with the LUMINOUS! Center of Excellence for Semiconductor Lighting and Displays, Nanyang

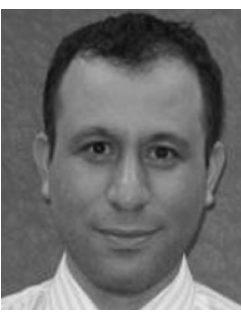
Technological University, Singapore. He has been a Postdoctoral Researcher with the National Nanotechnology Research Center (UNAM), Bilkent University, Ankara, Turkey, since 2019. He has published over $30 \mathrm{SCl}$ journal articles. He delivered his work at several international conferences as contributed/invited talks. His current research interests include the synthesis of colloidal quantum dots, nanoplatelets and magnetic nanoparticles, and optical and magneto-optical characterization of nanostructures. 
Hilmi Volkan Demir received the B.S. degree from Bilkent University, Ankara, Turkey, in 1998, and the M.S. and Ph.D. degrees from Stanford University, Stanford, CA, USA, in 2000 and 2004, respectively.

As Singapore's NRF Fellow, he is currently a Professor of electrical engineering, physics and materials with Nanyang Technological University (NTU), Singapore, where he is also the Director of the LUMINOUS! Center of Excellence for Semiconductor Lighting and Displays. Concurrently, he holds appointment at Bilkent University UNAM (his alma mater). His current research interests include semiconductor nanocrystal optoelectronics. He has published over $340 \mathrm{SCl}$ articles, delivered over 250 invited seminars/lectures/talks, and generated over 40 patents.
Dr. Demir is an elected Associate Member of the Turkish National Academy of Sciences (TUBA). These scientific and entrepreneurship activities resulted in important international and national awards, including the NRF Investigatorship Award, the Nanyang Award for Research Excellence, the European Science Foundation EURYI Award, the TUBITAK TESVIK Award, and the TUBA-GEBIP Award. He has been selected The Outstanding Young Person in the World (TOYP Award) of Junior Chamber International (JCl) Federation of Young Leaders and Entrepreneurs Worldwide in the category of academic achievement and leadership. $\mathrm{He}$ is currently the Springer-Nature Series Editor of Nanoscience and Nanotechnology and an OSA Editor of Optics Express. He served as Technical Chair in 2015, Member-at-Large in 2016, and General Chair in 2017, for the IEEE Photonics Society's flagship program IEEE Photonics Conference (IPC). 